\title{
Fuzzy fixed point results and applications to ordinary fuzzy differential equations in complex valued metric spaces
}

\author{
Muhammad Sarwar*1 (D), Humaira ${ }^{1}$ (D), Tongxing Li $^{2,3}$ (iD \\ ${ }^{1}$ Department of Mathematics, University of Malakand, Chakdara Dir(L), Khyber Pakhtunkhwa, Pakistan \\ ${ }^{2}$ School of Information Science and Engineering, Linyi University, Linyi, Shandong 276005, P. R. China \\ ${ }^{3}$ LinDa Institute of Shandong Provincial Key Laboratory of Network Based Intelligent Computing, Linyi \\ University, Linyi, Shandong 276005, P. R. China
}

\begin{abstract}
The main purpose of this article is to discuss the existence of the common solution of second-order nonlinear boundary value problems

$$
\left\{\begin{array}{l}
\mathfrak{x}^{\prime \prime}(\jmath)=\mathbb{k}\left(\jmath, \mathfrak{x}(\jmath), \mathfrak{x}^{\prime}(\jmath)\right), \quad \text { if } \jmath \in[0, \Lambda], \quad \Lambda>0, \\
\mathfrak{x}\left(\jmath_{1}\right)=\mathfrak{x}_{1}, \\
\mathfrak{x}\left(\jmath_{2}\right)=\mathfrak{x}_{2}, \quad \jmath_{1}, \jmath_{2} \in[0, \Lambda],
\end{array}\right.
$$

where $\mathbb{k}:[0, \Lambda] \times \mathfrak{S}(\mathcal{S}) \times \mathfrak{S}(\mathcal{S}) \rightarrow \mathfrak{S}(\mathcal{S})$ is a continuous function and $\mathfrak{S}(\mathcal{S})$ is a family of fuzzy sets. In this regard we obtain common fixed point results for two pairs of fuzzy mappings satisfying rational contractive condition in the setting of complex valued metric spaces. Our results improve those reported in the existing literature.
\end{abstract}

Mathematics Subject Classification (2010). 34A08, 34B37, 35R11

Keywords. complex valued metric space, fuzzy mapping, fixed point, common fixed point, Cauchy sequence, contractive condition

\section{Introduction}

Differential equations play a significant role in mathematical analysis and have numerous applications in the real world problems. The problems arise in different fields of sciences like physics, economics, engineering, and applied mathematics lead to differential equations which describe mathematical models. Somehow when the information arise from the computational perception is vague, uncertain and partially true or without sharp boundaries, researchers utilize the concept of fuzziness and describe mathematical models by fuzzy differential equations or fuzzy linear systems. In [1], the authors have derived a weak fuzzy solution for the system of fuzzy linear equations, while Salahshour et al. [30] proposed a technique to find the approximate solution of fully fuzzy linear system. There are various developed techniques to investigate the existence of the solution of differential

\footnotetext{
*Corresponding Author.

Email addresses: sarwarswati@gmail.com (M. Sarwar), humaira.swatpk@gmail.com (Humaira), litongx2007@163.com (T. Li)

Received: 22.12.2017; Accepted: 25.06.2018
} 
equations. Fixed point technique is one of the most powerful tools for obtaining the required task. We refer the reader to $[8,13,14,18,22-27,32,36,38]$ in which the researchers have used fixed point results to investigate the existence of the solution of mathematical models.

Banach contraction principle has been considered as the fundamental result of metric fixed point theory [7]. Several generalizations of Banach contraction principle have been demonstrated by many mathematicians in different frameworks like $G$ metric spaces, rectangular cone metric spaces, probabilistic metric, quasi metric and cone metric spaces; see $[9,11,19,29,31]$ for details.

Heilpern [15] has highly contributed in fuzzy set theory by introducing fuzzy mappings. He demonstrated the Banach contraction principle for fuzzy mapping in metric linear spaces and derived fixed point results in the said framework. Several generalizations of Heilpern's fixed point results have been derived by researchers in different spaces; see $[3,4,6,12,37]$. Recently, a variety of work has been done in solving various problems of many applied fields of mathematics by fuzzy polynomial. Jafarian and his co-authors designed a neural network to find the solution of fully fuzzy polynomial with degree $n$ in [16].

Dass and Gupta [10] generalized Banach contraction principle for rational type contractive inequality in metric space and addressed some fixed point results which are further generalized in various spaces by extending the contraction condition. In the meanwhile it was shown to be invalid approach to find fixed point results in vector spaces. They realized that rational type contractive condition fails where division of vectors occurs. Due to this defect the application of rational contractive condition does not work in cone metric spaces.

In this regard Azam et al. [5] introduced a notion of complex valued metric space which is a special case of cone metric spaces and obtained common fixed point results with rational contraction. Afterwards several researchers extended the aforesaid work using different types of rational contraction with self mappings and multivalued mappings in the context of complex valued metric spaces; see, for instance, $[2,17,20,21,34]$ and the references cited therein. The researchers continued the process of more generalizations and improved the contractive conditions by replacing control function to the Lipschitz constant in contraction. In addition Sintunavarat et al. [33,35], have applied some common fixed point results to investigate common solution to Urysohn integral equations in the context of complex valued metric spaces .

In this paper, we prove some common fixed point results for two pairs of fuzzy mappings satisfying a contractive condition of rational type in the setting of complex valued metric spaces. The derived results generalize some results in the context of complex valued metric space with fuzzy mappings.

As an application, we prove the existence of common solution of second-order nonlinear boundary value problems (0.1).

\section{Preliminaries}

Definition 2.1 ([5]). Let the set of complex numbers be denoted by $\complement$. For $\mathfrak{x}, \mathfrak{y} \in \complement$ we define a partial order $\precsim$ on $\complement$ such that:

$(\mathrm{Ci}) \quad \mathfrak{x} \precsim \mathfrak{y} \Longleftrightarrow \operatorname{Re}(\mathfrak{x}) \leq \operatorname{Re}(\mathfrak{y})$ and $\operatorname{Im}(\mathfrak{x}) \leq \operatorname{Im}(\mathfrak{y}) ;$

(Cii) $\mathfrak{x} \prec \mathfrak{y} \Longleftrightarrow \operatorname{Re}(\mathfrak{x})<\operatorname{Re}(\mathfrak{y})$ and $\operatorname{Im}(\mathfrak{x})<\operatorname{Im}(\mathfrak{y})$;

(Ciii) $\mathfrak{x} \precsim \mathfrak{y} \Longleftrightarrow \operatorname{Re}(\mathfrak{x})=\operatorname{Re}(\mathfrak{y})$ and $\operatorname{Im}(\mathfrak{x})<\operatorname{Im}(\mathfrak{y}) ;$

(Civ) $\mathfrak{x}=\mathfrak{y} \Longleftrightarrow \operatorname{Re}(\mathfrak{x})=\operatorname{Re}(\mathfrak{y})$ and $\operatorname{Im}(\mathfrak{x})=\operatorname{Im}(\mathfrak{y})$.

Clearly if $c \leq d, \Rightarrow c \mathfrak{y} \precsim d \mathfrak{y}$, for all $c, d \in \mathfrak{R}$. Note that, if $\mathfrak{x} \neq \mathfrak{y}$ and one of (Ci), (Cii), and (Ciii) is satisfied, then $\mathfrak{x} \precsim \mathfrak{y}$ and we write $\mathfrak{x}=\mathfrak{y}$ if only (Civ) satisfies. Note that

i) $0 \precsim \mathfrak{x} \precsim \mathfrak{y} \Rightarrow|\mathfrak{x}|<|\mathfrak{y}|, \forall \mathfrak{x}, \mathfrak{y} \in \complement$; 
ii) $\mathfrak{x} \precsim \mathfrak{y}$ and $\mathfrak{y} \prec \mathfrak{y}_{3} \Rightarrow \mathfrak{x} \prec \mathfrak{y}_{3}$, for all $\mathfrak{x}, \mathfrak{y}, \mathfrak{y}_{3} \in \mathcal{C}$.

Definition 2.2 ([5]). Let $\mathcal{S}$ be a nonempty set and $\sigma: \mathcal{S} \times \mathcal{S} \rightarrow \complement$ be a mapping satisfies the conditions given below:

1) $0 \precsim \sigma(\mathfrak{x}, \mathfrak{y})$, for all $\mathfrak{x}, \mathfrak{y} \in \mathcal{S}$ and $\sigma(\mathfrak{x}, \mathfrak{y})=0$ if and only if $\mathfrak{x}=\mathfrak{y}$;

2) $\sigma(\mathfrak{x}, \mathfrak{y})=\sigma(\mathfrak{y}, \mathfrak{x})$, for all $\mathfrak{x}, \mathfrak{y} \in \mathcal{S}$;

3) $\sigma(\mathfrak{x}, \mathfrak{y}) \precsim \sigma\left(\mathfrak{x}, \mathfrak{x}_{1}\right)+\sigma\left(\mathfrak{x}_{1}, \mathfrak{y}\right)$, for all $\mathfrak{x}, \mathfrak{x}_{1}, \mathfrak{y} \in \mathcal{S}$.

Then $(\mathcal{S}, \sigma)$ is called a complex valued metric space.

Definition $2.3([5])$. A point $\mathfrak{x} \in \mathcal{S}$ is known as an interior point of a set $Z \subseteq \mathcal{S}$, if we find $0 \prec \epsilon \in \complement$ with

$$
\mathfrak{B}(\mathfrak{x}, \epsilon)=\{\mathfrak{y} \in \mathcal{S}: \sigma(\mathfrak{x}, \mathfrak{y}) \prec \epsilon\} \subseteq Z .
$$

A point $\mathfrak{x} \in Z$ is known as the limit point of $Z$, if there exists an open ball $\mathfrak{B}(\mathfrak{x}, \epsilon)$ with

$$
\mathfrak{B}(\mathfrak{x}, \epsilon) \cap(Z \backslash\{\mathfrak{x}\}) \neq \phi,
$$

where $0 \prec \epsilon \in \mathcal{C}$. A subset $Z$ of $\mathcal{S}$ is said to be open if every point of $Z$ is an interior point of $Z$. Furthermore, $Z$ is said to be closed if it contains all its limit points. The family

$$
\nabla=\{\mathfrak{B}(\mathfrak{x}, \epsilon): \mathfrak{x} \in \mathcal{S}, 0 \prec \epsilon\}
$$

is a sub-basis for a Hausdorff topology $\Im$ on $\mathcal{S}$.

Definition 2.4. Let $(\mathcal{S}, \sigma)$ be a complex valued metric space. Throughout this paper, we have denoted the family of all nonempty closed bounded subsets of complex valued metric space $\mathcal{S}$ by $C B(\mathcal{S})$. For $\nu \in \mathfrak{\complement}$, we represent

$$
s(\nu)=\{\mathfrak{x} \in \complement: \nu \preceq \mathfrak{x}\}
$$

and for $\mathfrak{y} \in \mathcal{S}$ and $\mho_{1} \in C B(\mathcal{S})$,

$$
s(\mathfrak{y}, c)=\cup_{c \in \mho_{1}} s(\sigma(\mathfrak{y}, c))=\cup_{c \in \mho_{1}}\{\mathfrak{x} \in \complement: \sigma(\mathfrak{y}, c) \preceq \mathfrak{x}\} .
$$

For $\mho_{2}, \mho_{1} \in C B(\mathcal{S})$, we denote

$$
s\left(\mho_{2}, \mho_{1}\right)=\left(\cap_{p \in \mho_{2}} s\left(p, \mho_{1}\right)\right) \cap\left(\cap_{q \in \mho_{1}} s\left(q, \mho_{2}\right)\right) .
$$

Let $\Psi: \mathcal{S} \rightarrow C B(\mathcal{S})$ be a multivalued mapping. For $\mathfrak{x} \in \mathcal{S}$ and $Q \in C B(\mathcal{S})$ we define

$$
\mathcal{W}_{\mathfrak{x}}(Q)=\{\sigma(\mathfrak{x}, q): q \in Q\} .
$$

Thus, for $\mathfrak{x}, \mathfrak{y} \in \mathcal{S}$,

$$
\mathcal{W}_{\mathfrak{x}}(\Psi \mathfrak{y})=\{\sigma(\mathfrak{x}, v): v \in \Psi \mathfrak{y}\} .
$$

Definition $2.5([2])$. Assume that $(\mathcal{S}, \sigma)$ is a complex valued metric space, and the fuzzy mapping $K_{1}: \mathcal{S} \rightarrow \mathfrak{S}(\mathcal{S})$ observes the greatest lower bound property (glb property) on $(\mathcal{S}, \sigma)$. Then for any $w \in \mathcal{S}$ and $\propto \in(0,1]$, the greatest lower bound of $\mathrm{W}_{w}\left(\left[K_{1} y\right]_{\propto}\right)$ exists in $\complement$ for all $w, y \in \mathcal{S}$. Here, we denote $\sigma\left(w,\left[K_{1} y\right]_{\propto}\right)$ by the glb of $\mathrm{W}_{w}\left(\left[K_{1} y\right]_{\propto}\right)$, i.e.,

$$
\sigma\left(w,\left[K_{1} y\right]_{\propto}\right)=\inf \left\{\sigma(w, u): u \in\left[K_{1} y\right]_{\propto}\right\} .
$$

Definition 2.6 ([5]). Assume that $\left\{\mathfrak{y}_{r}\right\}$ is a sequence in complex valued metric space and $\mathfrak{y} \in \mathcal{S}$. Then we say that $\mathfrak{y}$ is a limit point of $\left\{\mathfrak{y}_{r}\right\}$ if for each $0 \prec \epsilon \in \mathfrak{\complement}$ there exists an $r_{0} \in \mathcal{N}$ with $\sigma\left(\mathfrak{y}_{r}, \mathfrak{y}\right) \preceq \epsilon$ for all $r \succeq r_{0}$; mathematically, we write $\lim _{r \rightarrow \infty} \mathfrak{y}_{r}=\mathfrak{y}$.

Definition $2.7([35])$. Let $\mathfrak{x}_{n}$ be a sequence in complex valued metric space $(\mathcal{S}, \sigma)$.

i) A sequence $\mathfrak{x}_{n}$ is called a C-Cauchy sequence in $\mathcal{S}$ if for any $\epsilon \in \complement$ with $0 \prec \epsilon$, there exists an $n_{0} \in \mathcal{N}$ such that for all $m, n>n_{0}, \sigma\left(\mathfrak{x}_{n}, \mathfrak{x}_{m}\right) \prec \epsilon$.

ii) $(\mathcal{S}, \sigma)$ is said to be a C-complete complex valued metric space if every C-Cauchy sequence is convergent in $\mathcal{S}$. 
Definition 2.8. Let $(V, \sigma)$ be a metric linear space. If $\mathcal{L}_{\mho_{1}}: V \rightarrow[0,1]$, then $\mathcal{L}_{\mho_{1}}$ is called membership function and the function value $\mathcal{L}_{\mho_{1}}(u)$ is called the grade of membership of $u$ in $\mho_{1}$ for $u \in V$. The set containing $u$ and its grade of membership is called fuzzy set. For simplicity we mentioned $\mathcal{L}_{\mho_{1}}$ by $\mho_{1}$, then the $\propto$-level set of $\mho_{1}$ is denoted by $\left[\mho_{1}\right]_{\propto}$ and is defined as follows:

$$
\begin{gathered}
{\left[\mho_{1}\right]_{\propto}=\left\{u: \mho_{1}(u) \geq \propto\right\}, \quad \text { if } \propto \in(0,1],} \\
{\left[\mho_{1}\right]_{0}=\left\{\overline{\left.u: \mho_{1}(u)>0\right\}} .\right.}
\end{gathered}
$$

Definition 2.9. Assume that $\mathfrak{S}(\mathcal{S})$ is the family of all fuzzy sets in a metric space $\mathcal{S}$. For $\mho_{1}, \mho_{2} \in \mathfrak{S}(\mathcal{S}), \mho_{1} \subset \mho_{2}$ means $\mho_{1}(\mathfrak{x}) \leq \mho_{2}(\mathfrak{x})$ for each $\mathfrak{x} \in \mathcal{S}$.

Lemma 2.10 ([31]). Assume that $(\mathcal{S}, d)$ is a complex valued metric space.

i) Let $u, v \in$ C. If $u \preceq v$, then $s(u) \subset s(v)$.

ii) Suppose $\mathfrak{x} \in \mathcal{S}$ and $\mho_{2} \in \mathbb{N}(\mathcal{S})$. If $\delta \in s\left(\mathfrak{x}, \mho_{2}\right)$, then $\mathfrak{x} \in \mho_{2}$.

iii) Let $v \in \complement, \mho_{2}, \mho_{1} \in C B(\mathcal{S})$ and $\hbar \in \mho_{2}$. If $v \in s\left(\mho_{2}, \mho_{1}\right)$, then $v \in s\left(\hbar, \mho_{1}\right)$ for all $\hbar \in \mho_{2}$ or $v \in s\left(\mho_{2}, \ell\right)$ for all $\ell \in \mho_{1}$.

Definition 2.11 ([15]). Assume that $Y$ is a metric space and $\mathcal{S}$ is an arbitrary set. If $F: \mathcal{S} \rightarrow \mathfrak{S}(\mathcal{S})$, then $F$ is called a fuzzy mapping. A fuzzy mapping $F$ is a fuzzy subset on $\mathcal{S} \times Y$ with membership function $F(\mathfrak{x})(y)$. The function $F(\mathfrak{x})(y)$ is the grade of membership of $y$ in $F(\mathfrak{x})$.

Definition 2.12 ([12]). If $(\mathcal{S}, \sigma)$ is a complex valued metric space and $K_{1}, K_{2}: \mathcal{S} \rightarrow \mathfrak{S}(\mathcal{S})$ are fuzzy mappings, then the point $\mathfrak{y} \in \mathcal{S}$ is said to be a fuzzy fixed point of $K_{1}$ if $\mathfrak{y} \in$ $\left[K_{1} \mathfrak{y}\right]_{\propto}$, where $\propto \in[0,1]$ and a common fuzzy fixed point of $K_{1}, K_{2}$ if $\mathfrak{y} \in\left[K_{1} \mathfrak{y}\right]_{\propto} \cap\left[K_{2} \mathfrak{y}\right]_{\propto}$.

\section{Main results}

In this paper, we have used the notation, introduced in [33] as given below:

$$
\complement_{+}=\{\mathfrak{x} \in \complement: \mathfrak{x} \succeq 0\}
$$

and

$$
\Gamma=\left\{£: \complement_{+} \rightarrow[0,1):\left\{\mathfrak{x}_{n}\right\} \subseteq \complement_{+} \quad \text { with } £\left(\mathfrak{x}_{n}\right) \rightarrow 1 \Rightarrow \mathfrak{x}_{n} \rightarrow 0\right\} .
$$

Theorem 3.1. Let $(\mathcal{S}, \sigma)$ be a $C$-complete complex valued metric space, and $K_{1}, K_{2}, K_{3}, K_{4}$ : $\mathcal{S} \rightarrow \mathfrak{S}(\mathcal{S})$ are fuzzy mappings satisfying the glb property. Let $\delta_{1}, \delta_{2}, \delta_{3}: \complement_{+} \rightarrow[0,1)$ be given mappings such that, for each $\mathfrak{x} \in \mathcal{S}$ and $\propto \in(0,1]$, there exist $\left[K_{1} \mathfrak{x}\right]_{\propto},\left[K_{2} \mathfrak{x}\right]_{\propto},\left[K_{3} \mathfrak{x}\right]_{\propto},\left[K_{4} \mathfrak{x}\right]_{\propto}$ (nonempty closed bounded subsets of $\mathcal{S}$ ). Suppose that the following conditions hold:

i) $\delta_{1}(\mathfrak{x})+\delta_{2}(\mathfrak{x})+\delta_{3}(\mathfrak{x})<1$ and the mapping $£: \complement_{+} \rightarrow[0,1)$ is defined by

$$
\beta(\mathfrak{x})=\frac{\delta_{1}(\mathfrak{x})}{1-\delta_{2}(\mathfrak{x})}
$$

for all $\mathfrak{x} \in \complement_{+}, £ \in \Gamma$;

ii) for every $\mathfrak{y}, \mathfrak{x} \in \mathcal{S}$, we have

$$
\begin{aligned}
& \delta_{1}\left(\sigma\left(\left[K_{3} \mathfrak{x}\right]_{\propto},\left[K_{4} \mathfrak{y}\right]_{\propto}\right)\right) \sigma\left(\left[K_{3} \mathfrak{x}\right]_{\propto},\left[K_{4} \mathfrak{y}\right]_{\propto}\right) \\
& +\delta_{2}\left(\sigma\left(\left[K_{3} \mathfrak{x}\right]_{\propto},\left[K_{4} \mathfrak{y}\right]_{\propto}\right)\right) \frac{\sigma\left(\left[K_{1} \mathfrak{x}\right]_{\propto},\left[K_{3} \mathfrak{x}\right]_{\propto}\right) \sigma\left(\left[K_{2} \mathfrak{y}\right]_{\propto},\left[K_{4} \mathfrak{y}\right]_{\propto}\right)}{1+\sigma(\mathfrak{x}, \mathfrak{y})} \\
& +\delta_{3}\left(\sigma\left(\left[K_{3} \mathfrak{x}\right]_{\propto},\left[K_{4} \mathfrak{y}\right]_{\propto}\right)\right) \frac{\sigma\left(\left[K_{1} \mathfrak{x}\right]_{\propto},\left[K_{4} \mathfrak{y}\right]_{\propto}\right) \sigma\left(\left[K_{2} \mathfrak{y}\right]_{\propto},\left[K_{3} \mathfrak{x}\right]_{\propto}\right)}{1+\sigma(\mathfrak{x}, \mathfrak{y})} \\
& \in s\left(\left[K_{1} \mathfrak{x}\right]_{\propto},\left[K_{2} \mathfrak{y}\right]_{\propto}\right) .
\end{aligned}
$$

If $\left[K_{1}(\mathcal{S})\right]_{\propto},\left[K_{2}(\mathcal{S})\right]_{\propto},\left[K_{3}(\mathcal{S})\right]_{\propto},\left[K_{4}(\mathcal{S})\right]_{\propto}$ are closed sets and $\left[K_{1}(\mathcal{S})\right]_{\propto} \subseteq\left[K_{4}(\mathcal{S})\right]_{\propto}$ and $\left[K_{2}(\mathcal{S})\right]_{\propto} \subseteq\left[K_{3}(\mathcal{S})\right]_{\propto}$, then $K_{1}, K_{2}, K_{3}, K_{4}$ have a common fuzzy fixed point in $\mathcal{S}$. 
Proof. Let $\mathfrak{x}_{0}$ be an arbitrary point in $\mathcal{S}$. Since $\left[K_{1}(\mathcal{S})\right]_{\propto} \subseteq\left[K_{4}(\mathcal{S})\right]_{\propto}$ and $\left[K_{2}(\mathcal{S})\right]_{\propto} \subseteq$ $\left[K_{3}(\mathcal{S})\right]_{\propto}$, we can define sequences $\left\{\mathfrak{x}_{n}\right\}$ and $\left\{\mathfrak{y}_{n}\right\}$ in $\mathcal{S}$ by $\mathfrak{y}_{2 n} \in\left[K_{1} \mathfrak{x}_{2 n-1}\right]_{\propto} \subseteq\left[K_{4} \mathfrak{x}_{2 n}\right]_{\propto}$ and $\mathfrak{y}_{2 n+1} \in\left[K_{2} \mathfrak{x}_{2 n}\right]_{\propto} \subseteq\left[K_{3} \mathfrak{x}_{2 n+1}\right]_{\propto}$ for all $n \in \mathcal{N}$. To prove that $\left\{\mathfrak{y}_{n}\right\}$ is a C-Cauchy sequence in $\mathcal{S}$, consider assumption (3.1) of Theorem 3.1 such that $\mathfrak{x}=\mathfrak{x}_{2 n-1}$ and $\mathfrak{y}=\mathfrak{x}_{2 n}$. Then

$$
\begin{aligned}
& \delta_{1}\left(\sigma\left(\left[K_{3} \mathfrak{x}_{2 n-1}\right]_{\propto},\left[K_{4} \mathfrak{x}_{2 n}\right]_{\propto}\right)\right) \sigma\left(\left[K_{3} \mathfrak{x}_{2 n-1}\right]_{\propto},\left[K_{4} \mathfrak{x}_{2 n}\right]_{\propto}\right) \\
& +\delta_{2}\left(\sigma\left(\left[K_{3} \mathfrak{x}_{2 n-1}\right]_{\propto},\left[K_{4} \mathfrak{x}_{2 n}\right]_{\propto}\right)\right) \frac{\sigma\left(\left[K_{1} \mathfrak{x}_{2 n-1}\right]_{\propto},\left[K_{3} \mathfrak{x}_{2 n-1}\right]_{\propto}\right) \sigma\left(\left[K_{2} \mathfrak{x}_{2 n}\right]_{\propto},\left[K_{4} \mathfrak{x}_{2 n}\right]_{\propto}\right)}{1+\sigma\left(\mathfrak{x}_{2 n-1}, \mathfrak{x}_{2 n}\right)} \\
& +\delta_{3}\left(\sigma\left(\left[K_{3} \mathfrak{x}_{2 n-1}\right]_{\propto},\left[K_{4} \mathfrak{x}_{2 n}\right]_{\propto}\right)\right) \frac{\sigma\left(\left[K_{1} \mathfrak{x}_{2 n-1}\right]_{\propto},\left[K_{4} \mathfrak{x}_{2 n}\right]_{\propto}\right) \sigma\left(\left[K_{2} \mathfrak{x}_{2 n}\right]_{\propto},\left[K_{3} \mathfrak{x}_{2 n-1}\right]_{\propto}\right)}{1+\sigma\left(\mathfrak{x}_{2 n-1}, \mathfrak{x}_{2 n}\right)} \\
& \in s\left(\left[K_{1} \mathfrak{x}_{2 n-1}\right]_{\propto},\left[K_{2} \mathfrak{x}_{2 n}\right]_{\propto}\right) .
\end{aligned}
$$

It follows from $\mathfrak{y}_{2 n} \in\left[K_{1} \mathfrak{x}_{2 n-1}\right]_{\propto}$ and Lemma 2.10 (iii) that

$$
\begin{aligned}
& \delta_{1}\left(\sigma\left(\left[K_{3} \mathfrak{x}_{2 n-1}\right]_{\propto},\left[K_{4} \mathfrak{x}_{2 n}\right]_{\propto}\right)\right) \sigma\left(\left[K_{3} \mathfrak{x}_{2 n-1}\right]_{\propto},\left[K_{4} \mathfrak{x}_{2 n}\right]_{\propto}\right) \\
& +\delta_{2}\left(\sigma\left(\left[K_{3} \mathfrak{x}_{2 n-1}\right]_{\propto},\left[K_{4} \mathfrak{x}_{2 n}\right]_{\propto}\right)\right) \frac{\sigma\left(\left[K_{1} \mathfrak{x}_{2 n-1}\right]_{\propto},\left[K_{3} \mathfrak{x}_{2 n-1}\right]_{\propto}\right) \sigma\left(\left[K_{2} \mathfrak{x}_{2 n}\right]_{\propto},\left[K_{4} \mathfrak{x}_{2 n}\right]_{\propto}\right)}{1+\sigma\left(\mathfrak{x}_{2 n-1}, \mathfrak{x}_{2 n}\right)} \\
& +\delta_{3}\left(\sigma\left(\left[K_{3} \mathfrak{x}_{2 n-1}\right]_{\propto},\left[K_{4} \mathfrak{x}_{2 n}\right]_{\propto}\right)\right) \frac{\sigma\left(\left[K_{1} \mathfrak{x}_{2 n-1}\right]_{\propto},\left[K_{4} \mathfrak{x}_{2 n}\right]_{\propto}\right) \sigma\left(\left[K_{2} \mathfrak{x}_{2 n}\right]_{\propto},\left[K_{3} \mathfrak{x}_{2 n-1}\right]_{\propto}\right)}{1+\sigma\left(\mathfrak{x}_{2 n-1}, \mathfrak{x}_{2 n}\right)} \\
& \in s\left(\mathfrak{y}_{2 n},\left[K_{2} \mathfrak{x}_{2 n}\right]_{\propto}\right) .
\end{aligned}
$$

Since $\left[K_{2} \mathfrak{x}_{2 n}\right]_{\propto}$ is a nonempty bounded closed set, there exists some $\mathfrak{y}_{2 n+1} \in\left[K_{2} \mathfrak{x}_{2 n}\right]_{\propto}$ with

$$
\begin{aligned}
& \delta_{1}\left(\sigma\left(\left[K_{3} \mathfrak{x}_{2 n-1}\right]_{\propto},\left[K_{4} \mathfrak{x}_{2 n}\right]_{\propto}\right)\right) \sigma\left(\left[K_{3} \mathfrak{x}_{2 n-1}\right]_{\propto},\left[K_{4} \mathfrak{x}_{2 n}\right]_{\propto}\right) \\
& +\delta_{2}\left(\sigma\left(\left[K_{3} \mathfrak{x}_{2 n-1}\right]_{\propto},\left[K_{4} \mathfrak{x}_{2 n}\right]_{\propto}\right)\right) \frac{\sigma\left(\left[K_{1} \mathfrak{x}_{2 n-1}\right]_{\propto},\left[K_{3} \mathfrak{x}_{2 n-1}\right]_{\propto}\right) \sigma\left(\left[K_{2} \mathfrak{x}_{2 n}\right]_{\propto},\left[K_{4} \mathfrak{x}_{2 n}\right]_{\propto}\right)}{1+\sigma\left(\mathfrak{x}_{2 n-1}, \mathfrak{x}_{2 n}\right)} \\
& +\delta_{3}\left(\sigma\left(\left[K_{3} \mathfrak{x}_{2 n-1}\right]_{\propto},\left[K_{4} \mathfrak{x}_{2 n}\right]_{\propto}\right)\right) \frac{\sigma\left(\left[K_{1} \mathfrak{x}_{2 n-1}\right]_{\propto},\left[K_{4} \mathfrak{x}_{2 n}\right]_{\propto}\right) \sigma\left(\left[K_{2} \mathfrak{x}_{2 n}\right]_{\propto},\left[K_{3} \mathfrak{x}_{2 n-1}\right]_{\propto}\right)}{1+\sigma\left(\mathfrak{x}_{2 n-1}, \mathfrak{x}_{2 n}\right)} \\
& \in s\left(\sigma\left(\mathfrak{y}_{2 n}, \mathfrak{y}_{2 n+1}\right)\right),
\end{aligned}
$$

that is,

$$
\begin{aligned}
\sigma\left(\mathfrak{y}_{2 n}, \mathfrak{y}_{2 n+1}\right) \preceq \quad & \delta_{1}\left(\sigma\left(\left[K_{3} \mathfrak{x}_{2 n-1}\right]_{\propto},\left[K_{4} \mathfrak{x}_{2 n}\right]_{\propto}\right)\right) \sigma\left(\left[K_{3} \mathfrak{x}_{2 n-1}\right]_{\propto},\left[K_{4} \mathfrak{x}_{2 n}\right]_{\propto}\right) \\
+ & \delta_{2}\left(\sigma\left(\left[K_{3} \mathfrak{x}_{2 n-1}\right]_{\propto},\left[K_{4} \mathfrak{x}_{2 n}\right]_{\propto}\right)\right) \\
& \times \frac{\sigma\left(\left[K_{1} \mathfrak{x}_{2 n-1}\right]_{\propto},\left[K_{3} \mathfrak{x}_{2 n-1}\right]_{\propto}\right) \sigma\left(\left[K_{2} \mathfrak{x}_{2 n}\right]_{\propto},\left[K_{4} \mathfrak{x}_{2 n}\right]_{\propto}\right)}{1+\sigma\left(\mathfrak{x}_{2 n-1}, \mathfrak{x}_{2 n}\right)} \\
+ & \delta_{3}\left(\sigma\left(\left[K_{3} \mathfrak{x}_{2 n-1}\right]_{\propto},\left[K_{4} \mathfrak{x}_{2 n}\right]_{\propto}\right)\right) \\
& \times \frac{\sigma\left(\left[K_{1} \mathfrak{x}_{2 n-1}\right]_{\propto},\left[K_{4} \mathfrak{x}_{2 n}\right]_{\propto}\right) \sigma\left(\left[K_{2} \mathfrak{x}_{2 n}\right]_{\propto},\left[K_{3} \mathfrak{x}_{2 n-1}\right]_{\propto}\right)}{1+\sigma\left(\mathfrak{x}_{2 n-1}, \mathfrak{x}_{2 n}\right)} .
\end{aligned}
$$


Using the glb property, we conclude that

$$
\begin{aligned}
\sigma\left(\mathfrak{y}_{2 n}, \mathfrak{y}_{2 n+1}\right) \preceq & \delta_{1}\left(\sigma\left(\mathfrak{y}_{2 n-1}, \mathfrak{y}_{2 n}\right)\right) \sigma\left(\mathfrak{y}_{2 n-1}, \mathfrak{y}_{2 n}\right) \\
& +\delta_{2}\left(\sigma\left(\mathfrak{y}_{2 n-1}, \mathfrak{y}_{2 n}\right)\right) \frac{\sigma\left(\mathfrak{y}_{2 n}, \mathfrak{y}_{2 n-1}\right) \sigma\left(\mathfrak{y}_{2 n+1}, \mathfrak{y}_{2 n}\right)}{1+\sigma\left(\mathfrak{y}_{2 n-1}, \mathfrak{y}_{2 n}\right)} \\
& +\delta_{3}\left(\sigma\left(\mathfrak{y}_{2 n-1}, \mathfrak{y}_{2 n}\right)\right) \frac{\sigma\left(\mathfrak{y}_{2 n}, \mathfrak{y}_{2 n}\right) \sigma\left(\mathfrak{y}_{2 n+1}, \mathfrak{y}_{2 n}\right)}{1+\sigma\left(\mathfrak{y}_{2 n-1}, \mathfrak{y}_{2 n}\right)} \\
\preceq & \delta_{1}\left(\sigma\left(\mathfrak{y}_{2 n-1}, \mathfrak{y}_{2 n}\right)\right) \sigma\left(\mathfrak{y}_{2 n-1}, \mathfrak{y}_{2 n}\right) \\
& +\delta_{2}\left(\sigma\left(\mathfrak{y}_{2 n-1}, \mathfrak{y}_{2 n}\right)\right) \sigma\left(\mathfrak{y}_{2 n+1}, \mathfrak{y}_{2 n}\right) \\
\preceq & \frac{\delta_{1}\left(\sigma\left(\mathfrak{y}_{2 n-1}, \mathfrak{y}_{2 n}\right)\right)}{1-\delta_{2}\left(\sigma\left(\mathfrak{y}_{2 n-1}, \mathfrak{y}_{2 n}\right)\right)} \sigma\left(\mathfrak{y}_{2 n-1}, \mathfrak{y}_{2 n}\right) \\
\Rightarrow\left|\sigma\left(\mathfrak{y}_{2 n}, \mathfrak{y}_{2 n+1}\right)\right| \leq & \frac{\delta_{1}\left(\sigma\left(\mathfrak{y}_{2 n-1}, \mathfrak{y}_{2 n}\right)\right)}{1-\delta_{2}\left(\sigma\left(\mathfrak{y}_{2 n-1}, \mathfrak{y}_{2 n}\right)\right)}\left|\sigma\left(\mathfrak{y}_{2 n-1}, \mathfrak{y}_{2 n}\right)\right|
\end{aligned}
$$

for all $n \in \mathcal{N}$. Utilizing condition (i) of Theorem 3.1, we get

$$
\left|\sigma\left(\mathfrak{y}_{2 n}, \mathfrak{y}_{2 n+1}\right)\right| \leq £\left(\sigma\left(\mathfrak{y}_{2 n-1}, \mathfrak{y}_{2 n}\right)\right)\left|\sigma\left(\mathfrak{y}_{2 n-1}, \mathfrak{y}_{2 n}\right)\right| .
$$

For $\mathfrak{y}_{2 n+1} \in\left[K_{2} \mathfrak{x}_{2 n}\right]_{\propto}$,

$$
\begin{aligned}
& \delta_{1}\left(\sigma\left(\left[K_{3} \mathfrak{x}_{2 n+1}\right]_{\propto},\left[K_{4} \mathfrak{x}_{2 n}\right]_{\propto}\right)\right) \sigma\left(\left[K_{3} \mathfrak{x}_{2 n+1}\right]_{\propto},\left[K_{4} \mathfrak{x}_{2 n}\right]_{\propto}\right) \\
& +\delta_{2}\left(\sigma\left(\left[K_{3} \mathfrak{x}_{2 n+1}\right]_{\propto},\left[K_{4} \mathfrak{x}_{2 n}\right]_{\propto}\right)\right) \frac{\sigma\left(\left[K_{1} \mathfrak{x}_{2 n+1}\right]_{\propto},\left[K_{3} \mathfrak{x}_{2 n+1}\right]_{\propto}\right) \sigma\left(\left[K_{2} \mathfrak{x}_{2 n}\right]_{\propto},\left[K_{4} \mathfrak{x}_{2 n}\right]_{\propto}\right)}{1+\sigma\left(\mathfrak{x}_{2 n+1}, \mathfrak{x}_{2 n}\right)} \\
& +\delta_{3}\left(\sigma\left(\left[K_{3} \mathfrak{x}_{2 n+1}\right]_{\propto},\left[H \mathfrak{x}_{2 n}\right]_{\propto}\right)\right) \frac{\sigma\left(\left[K_{1} \mathfrak{x}_{2 n+1}\right]_{\propto},\left[K_{4} \mathfrak{x}_{2 n}\right]_{\propto}\right) \sigma\left(\left[K_{2} \mathfrak{x}_{2 n}\right]_{\propto},\left[K_{3} \mathfrak{x}_{2 n+1}\right]_{\propto}\right)}{1+\sigma\left(\mathfrak{x}_{2 n+1}, \mathfrak{x}_{2 n}\right)} \\
& \in s\left(\left[K_{1} \mathfrak{x}_{2 n+1}\right]_{\propto},\left[K_{2} \mathfrak{x}_{2 n}\right]_{\propto}\right) .
\end{aligned}
$$

Using Lemma 2.10 (iii), we have

$$
\begin{aligned}
& \delta_{1}\left(\sigma\left(\left[K_{3} \mathfrak{x}_{2 n+1}\right]_{\propto},\left[K_{4} \mathfrak{x}_{2 n}\right]_{\propto}\right)\right) \sigma\left(\left[K_{3} \mathfrak{x}_{2 n+1}\right]_{\propto},\left[K_{4} \mathfrak{x}_{2 n}\right]_{\propto}\right) \\
& +\delta_{2}\left(\sigma\left(\left[K_{3} \mathfrak{x}_{2 n+1}\right]_{\propto},\left[K_{4} \mathfrak{x}_{2 n}\right]_{\propto}\right)\right) \frac{\sigma\left(\left[K_{1} \mathfrak{x}_{2 n+1}\right]_{\propto},\left[K_{3} \mathfrak{x}_{2 n+1}\right]_{\propto}\right) \sigma\left(\left[K_{2} \mathfrak{x}_{2 n}\right]_{\propto},\left[K_{4} \mathfrak{x}_{2 n}\right]_{\propto}\right)}{1+\sigma\left(\mathfrak{x}_{2 n+1}, \mathfrak{x}_{2 n}\right)} \\
& +\delta_{3}\left(\sigma\left(\left[K_{3} \mathfrak{x}_{2 n+1}\right]_{\propto},\left[K_{4} \mathfrak{x}_{2 n}\right]_{\propto}\right)\right) \frac{\sigma\left(\left[E \mathfrak{x}_{2 n+1}\right]_{\propto},\left[K_{4} \mathfrak{x}_{2 n}\right]_{\propto}\right) \sigma\left(\left[K_{2} \mathfrak{x}_{2 n}\right]_{\propto},\left[K_{3} \mathfrak{x}_{2 n+1}\right]_{\propto}\right)}{1+\sigma\left(\mathfrak{x}_{2 n+1}, \mathfrak{x}_{2 n}\right)} \\
& \in s\left(\left[K_{1} \mathfrak{x}_{2 n+1}\right]_{\propto}, \mathfrak{y}_{2 n+1}\right) .
\end{aligned}
$$

Then there exists a $w_{2 n+2} \in\left[K_{1} \mathfrak{x}_{2 n+1}\right]_{\propto}$ such that

$$
\begin{aligned}
& \delta_{1}\left(\sigma\left(\left[K_{3} \mathfrak{x}_{2 n+1}\right]_{\propto},\left[K_{4} \mathfrak{x}_{2 n}\right]_{\propto}\right)\right) \sigma\left(\left[K_{3} \mathfrak{x}_{2 n+1}\right]_{\propto},\left[K_{4} \mathfrak{x}_{2 n}\right]_{\propto}\right) \\
& +\delta_{2}\left(\sigma\left(\left[K_{3} \mathfrak{x}_{2 n+1}\right]_{\propto},\left[K_{4} \mathfrak{x}_{2 n}\right]_{\propto}\right)\right) \frac{\sigma\left(\left[K_{1} \mathfrak{x}_{2 n+1}\right]_{\propto},\left[K_{3} \mathfrak{x}_{2 n+1}\right]_{\propto}\right) \sigma\left(\left[K_{2} \mathfrak{x}_{2 n}\right]_{\propto},\left[K_{4} \mathfrak{x}_{2 n}\right]_{\propto}\right)}{1+\sigma\left(\mathfrak{x}_{2 n+1}, \mathfrak{x}_{2 n}\right)} \\
& +\delta_{3}\left(\sigma\left(\left[K_{3} \mathfrak{x}_{2 n+1}\right]_{\propto},\left[K_{4} \mathfrak{x}_{2 n}\right]_{\propto}\right)\right) \frac{\sigma\left(\left[K_{1} \mathfrak{x}_{2 n+1}\right]_{\propto},\left[K_{4} \mathfrak{x}_{2 n}\right]_{\propto}\right) \sigma\left(\left[K_{2} \mathfrak{x}_{2 n}\right]_{\propto},\left[K_{3} \mathfrak{x}_{2 n+1}\right]_{\propto}\right)}{1+\sigma\left(\mathfrak{x}_{2 n+1}, \mathfrak{x}_{2 n}\right)} \\
& \in s\left(\sigma\left(\mathfrak{y}_{2 n+2}, \mathfrak{y}_{2 n+1}\right)\right)
\end{aligned}
$$


that is,

$$
\begin{aligned}
\sigma\left(\mathfrak{y}_{2 n+2}, \mathfrak{y}_{2 n+1}\right) \preceq \quad & \delta_{1}\left(\sigma\left(\left[K_{3} \mathfrak{x}_{2 n+1}\right]_{\propto},\left[K_{4} \mathfrak{x}_{2 n}\right]_{\propto}\right)\right) \sigma\left(\left[K_{3} \mathfrak{x}_{2 n+1}\right]_{\propto},\left[K_{4} \mathfrak{x}_{2 n}\right]_{\propto}\right) \\
+ & \delta_{2}\left(\sigma\left(\left[K_{3} \mathfrak{x}_{2 n+1}\right]_{\propto},\left[K_{4} \mathfrak{x}_{2 n}\right]_{\propto}\right)\right) \\
& \times \frac{\sigma\left(\left[K_{1} \mathfrak{x}_{2 n+1}\right]_{\propto},\left[K_{3} \mathfrak{x}_{2 n+1}\right]_{\propto}\right) \sigma\left(\left[K_{2} \mathfrak{x}_{2 n}\right]_{\propto},\left[K_{4} \mathfrak{x}_{2 n}\right]_{\propto}\right)}{1+\sigma\left(\mathfrak{x}_{2 n+1}, \mathfrak{x}_{2 n}\right)} \\
+ & \delta_{3}\left(\sigma\left(\left[K_{3} \mathfrak{x}_{2 n+1}\right]_{\propto},\left[K_{4} \mathfrak{x}_{2 n}\right]_{\propto}\right)\right) \\
& \times \frac{\sigma\left(\left[K_{1} \mathfrak{x}_{2 n+1}\right]_{\propto},\left[K_{4} \mathfrak{x}_{2 n}\right]_{\propto}\right) \sigma\left(\left[K_{2} \mathfrak{x}_{2 n}\right]_{\propto},\left[K_{3} \mathfrak{x}_{2 n+1}\right]_{\propto}\right)}{1+\sigma\left(\mathfrak{x}_{2 n+1}, \mathfrak{x}_{2 n}\right)} .
\end{aligned}
$$

An application of glb property implies that

$$
\begin{aligned}
\sigma\left(\mathfrak{y}_{2 n+2}, \mathfrak{y}_{2 n+1}\right) \preceq & \delta_{1}\left(\sigma\left(\mathfrak{y}_{2 n+1}, \mathfrak{y}_{2 n}\right)\right) \sigma\left(\mathfrak{y}_{2 n+1}, \mathfrak{y}_{2 n}\right) \\
& +\delta_{2}\left(\sigma\left(\mathfrak{y}_{2 n+1}, \mathfrak{y}_{2 n}\right)\right) \frac{\sigma\left(\mathfrak{y}_{2 n+2}, \mathfrak{y}_{2 n+1}\right) \sigma\left(\mathfrak{y}_{2 n+1}, \mathfrak{y}_{2 n}\right)}{1+\sigma\left(\mathfrak{y}_{2 n+1}, \mathfrak{y}_{2 n}\right)} \\
& +\delta_{3}\left(\sigma\left(\mathfrak{y}_{2 n+1}, \mathfrak{y}_{2 n}\right)\right) \frac{\sigma\left(\mathfrak{y}_{2 n+2}, \mathfrak{y}_{2 n}\right) \sigma\left(\mathfrak{y}_{2 n+1}, \mathfrak{y}_{2 n+1}\right)}{1+\sigma\left(\mathfrak{y}_{2 n+1}, \mathfrak{y}_{2 n}\right)} \\
\preceq & \delta_{1}\left(\sigma\left(\mathfrak{y}_{2 n+1}, \mathfrak{y}_{2 n}\right)\right) \sigma\left(\mathfrak{y}_{2 n+1}, \mathfrak{y}_{2 n}\right) \\
& +\delta_{2}\left(\sigma\left(\mathfrak{y}_{2 n+1}, \mathfrak{y}_{2 n}\right)\right) \sigma\left(\mathfrak{y}_{2 n+2}, \mathfrak{y}_{2 n+1}\right) \\
\preceq & \frac{\delta_{1}\left(\sigma\left(\mathfrak{y}_{2 n+1}, \mathfrak{y}_{2 n}\right)\right)}{1-\delta_{2}\left(\sigma\left(\mathfrak{y}_{2 n+1}, \mathfrak{y}_{2 n}\right)\right)} \sigma\left(\mathfrak{y}_{2 n+1}, \mathfrak{y}_{2 n}\right) \\
\Rightarrow\left|\sigma\left(\mathfrak{y}_{2 n+2}, \mathfrak{y}_{2 n+1}\right)\right| \leq & \frac{\delta_{1}\left(\sigma\left(\mathfrak{y}_{2 n+1}, \mathfrak{y}_{2 n}\right)\right)}{1-\delta_{2}\left(\sigma\left(\mathfrak{y}_{2 n+1}, \mathfrak{y}_{2 n}\right)\right)}\left|\sigma\left(\mathfrak{y}_{2 n+1}, \mathfrak{y}_{2 n}\right)\right|
\end{aligned}
$$

for all $n \in \mathcal{N}$. Applying condition (i) of Theorem 3.1, we get

$$
\left|\sigma\left(\mathfrak{y}_{2 n+2}, \mathfrak{y}_{2 n+1}\right)\right| \leq £\left(\sigma\left(\mathfrak{y}_{2 n+1}, \mathfrak{y}_{2 n}\right)\right)\left|\sigma\left(\mathfrak{y}_{2 n+1}, \mathfrak{y}_{2 n}\right)\right| .
$$

Consequently, we obtain

$$
\left|\sigma\left(\mathfrak{y}_{n}, \mathfrak{y}_{n+1}\right)\right| \leq £\left(\sigma\left(\mathfrak{y}_{n-1}, \mathfrak{y}_{n}\right)\right)\left|\sigma\left(\mathfrak{y}_{n-1}, \mathfrak{y}_{n}\right)\right| \leq\left|\sigma\left(\mathfrak{y}_{n-1}, \mathfrak{y}_{n}\right)\right| .
$$

This implies that the sequence $\left\{\left|\mathfrak{y}_{n}, \mathfrak{y}_{n+1}\right|\right\}_{n \in \mathcal{N}}$ is a nonincreasing monotonic and bounded from below. Therefore, $\left|\mathfrak{y}_{n}, \mathfrak{y}_{n+1}\right| \rightarrow r$ for some $r \geq 0$. We will prove that $r=0$. For this, assuming $r>0$ and taking limit as $n \rightarrow \infty$ in (3.2), we deduce that

$$
1 \leq \lim _{n \rightarrow \infty} £\left(\sigma\left(\mathfrak{y}_{n-1}, \mathfrak{y}_{n}\right)\right) \leq 1 \Rightarrow \lim _{n \rightarrow \infty} £\left(\sigma\left(\mathfrak{y}_{n-1}, \mathfrak{y}_{n}\right)\right)=1 .
$$

Since $£ \in \Gamma$, we get $\left|\sigma\left(\mathfrak{y}_{n-1}, \mathfrak{y}_{n}\right)\right| \rightarrow 0$, which is a contradiction. Therefore, we have $r=0$, that is,

$$
\lim _{n \rightarrow \infty}\left|\sigma\left(\mathfrak{y}_{n-1}, \mathfrak{y}_{n}\right)\right|=0 .
$$

Next, we have to show that $\left\{\mathfrak{y}_{n}\right\}$ is a C-Cauchy sequence. It is enough to show that $\left\{\mathfrak{y}_{2 n}\right\}$ is a C-Cauchy sequence. Suppose on the contrary that $\left\{\mathfrak{y}_{2 n}\right\}$ is not a C-Cauchy sequence. Then, by using Definition 2.7, there exists an $\epsilon \in \complement$ such that $\epsilon \succ 0$, for which there exist $2 n_{q}>2 m_{q} \geq q$ for all $q \in \mathcal{N}$ such that

$$
\sigma\left(\mathfrak{y}_{2 n_{q}}, \mathfrak{y}_{2 m_{q}}\right) \succeq \epsilon .
$$

Furthermore, we can choose $m_{q}$ correspondingly to $n_{q}$ such that it is the smallest integer with $2 m_{q}>2 n_{q} \geq q$ satisfying (3.4). Then

$$
\sigma\left(\mathfrak{y}_{2 n_{q}}, \mathfrak{y}_{2 m_{q}-2}\right) \prec \epsilon .
$$


By (3.4), (3.5), and triangular inequality, we obtain

$$
\begin{aligned}
\epsilon & \preceq \sigma\left(\mathfrak{y}_{2 n_{q}}, \mathfrak{y}_{2 m_{q}}\right) \\
& \preceq \sigma\left(\mathfrak{y}_{2 n_{q}}, \mathfrak{y}_{2 m_{q}-2}\right)+\sigma\left(\mathfrak{y}_{2 m_{q}-2}, \mathfrak{y}_{2 m_{k}-1}\right)+\sigma\left(\mathfrak{y}_{2 m_{q}-1}, \mathfrak{y}_{2 m_{q}}\right) \\
& \preceq \epsilon+\sigma\left(\mathfrak{y}_{2 m_{q}-2}, \mathfrak{y}_{2 m_{q}-1}\right)+\sigma\left(\mathfrak{y}_{2 m_{q}-1}, \mathfrak{y}_{2 m_{q}}\right),
\end{aligned}
$$

which yields

$$
|\epsilon| \leq\left|\sigma\left(\mathfrak{y}_{2 n_{q}}, \mathfrak{y}_{2 m_{q}}\right)\right| \leq|\epsilon|+\left|\sigma\left(\mathfrak{y}_{2 m_{q}-2}, \mathfrak{y}_{2 m_{q}-1}\right)\right|+\left|\sigma\left(\mathfrak{y}_{2 m_{q}-1}, \mathfrak{y}_{2 m_{q}}\right)\right| .
$$

Taking limit $q \rightarrow \infty$ and using (3.3), we have

$$
|\epsilon| \leq \lim _{q \rightarrow \infty}\left|\sigma\left(\mathfrak{y}_{2 n_{q}}, \mathfrak{y}_{2 m_{q}}\right)\right| \leq|\epsilon| \Rightarrow \lim _{q \rightarrow \infty}\left|\sigma\left(\mathfrak{y}_{2 n_{q}}, \mathfrak{y}_{2 m_{q}}\right)\right|=|\epsilon| .
$$

Again, by triangular inequality, we get

$$
\begin{aligned}
\left|\sigma\left(\mathfrak{y}_{2 n_{q}}, \mathfrak{y}_{2 m_{q}}\right)\right| & \leq\left|\sigma\left(\mathfrak{y}_{2 n_{q}}, \mathfrak{y}_{2 m_{q}+1}\right)\right|+\left|\sigma\left(\mathfrak{y}_{2 m_{q}+1}, \mathfrak{y}_{2 m_{q}}\right)\right| \\
& \leq\left|\sigma\left(\mathfrak{y}_{2 n_{q}}, \mathfrak{y}_{2 m_{q}}\right)\right|+\left|\sigma\left(\mathfrak{y}_{2 m_{q}}, \mathfrak{y}_{2 m_{q}+1}\right)\right|+\left|\sigma\left(\mathfrak{y}_{2 m_{q}+1}, \mathfrak{y}_{2 m_{q}}\right)\right| .
\end{aligned}
$$

Letting $q \rightarrow \infty$ and applying (3.3) and (3.6) in the above two inequalities, we conclude that

$$
\lim _{q \rightarrow \infty}\left|\sigma\left(\mathfrak{y}_{2 n_{q}}, \mathfrak{y}_{2 m_{q}+1}\right)\right|=|\epsilon|
$$

Furthermore, we have

$$
\sigma\left(\mathfrak{y}_{2 n_{q}}, \mathfrak{y}_{2 m_{q}+1}\right) \preceq \sigma\left(\mathfrak{y}_{2 n_{q}}, \mathfrak{y}_{2 n_{q}+1}\right)+\sigma\left(\mathfrak{y}_{2 n_{q}+1}, \mathfrak{y}_{2 m_{q}+2}\right)+\sigma\left(\mathfrak{y}_{2 m_{q}+2}, \mathfrak{y}_{2 m_{q}+1}\right) .
$$

Consider $\sigma\left(\mathfrak{y}_{2 n_{q}+1}, \mathfrak{y}_{2 m_{q}+2}\right)$. By using condition (ii) of Theorem 3.1 with $\mathfrak{x}=\mathfrak{x}_{2 n_{q}}$ and $\mathfrak{y}=\mathfrak{x}_{2 m_{q}+1}$, we deduce that

$$
\begin{aligned}
& \delta_{1}\left(\sigma\left(\left[K_{3} \mathfrak{x}_{2 n_{q}}\right]_{\propto},\left[K_{4} \mathfrak{x}_{2 m_{q}+1}\right]_{\propto}\right)\right) \sigma\left(\left[K_{3} \mathfrak{x}_{2 n_{q}}\right]_{\propto},\left[K_{4} \mathfrak{x}_{2 m_{q}+1}\right]_{\propto}\right) \\
& +\delta_{2}\left(\sigma\left(\left[K_{3} \mathfrak{x}_{2 n_{q}}\right]_{\propto},\left[K_{4} \mathfrak{x}_{2 m_{q}+1}\right]_{\propto}\right)\right) \frac{\sigma\left(\left[K_{1} \mathfrak{x}_{2 n_{q}}\right]_{\propto},\left[K_{3} \mathfrak{x}_{2 n_{q}}\right]_{\propto}\right) \sigma\left(\left[K_{2} \mathfrak{x}_{2 m_{q}+1}\right]_{\propto},\left[K_{4} \mathfrak{x}_{2 m_{q}+1}\right]_{\propto}\right)}{1+\sigma\left(\mathfrak{x}_{2 n_{q}}, \mathfrak{x}_{2 m_{q}+1}\right)} \\
& +\delta_{3}\left(\sigma\left(\left[K_{3} \mathfrak{x}_{2 n_{q}}\right]_{\propto},\left[K_{4} \mathfrak{x}_{2 m_{q}+1}\right]_{\propto}\right)\right) \frac{\sigma\left(\left[K_{1} \mathfrak{x}_{2 n_{q}}\right]_{\propto},\left[K_{4} \mathfrak{x}_{2 m_{q}+1}\right]_{\propto}\right) \sigma\left(\left[K_{2} \mathfrak{x}_{2 m_{q}+1}\right]_{\propto},\left[K_{3} \mathfrak{x}_{2 n_{q}}\right]_{\propto}\right)}{1+\sigma\left(\mathfrak{x}_{2 n_{q}}, \mathfrak{x}_{2 m_{q}+1}\right)} \\
& \in s\left(\left[K_{1} \mathfrak{x}_{2 n_{q}}\right]_{\propto},\left[K_{2} \mathfrak{x}_{2 m_{q}+1}\right]_{\propto}\right) .
\end{aligned}
$$

Using construction of sequence, we obtain that $\mathfrak{y}_{2 n_{q}+1} \in\left[K_{1} \mathfrak{x}_{2 n_{q}}\right]_{\propto}$. Therefore, by virtue of Lemma 2.10 (iii), we arrive at

$$
\begin{aligned}
& \delta_{1}\left(\sigma\left(\left[K_{3} \mathfrak{x}_{2 n_{q}}\right]_{\propto},\left[K_{4} \mathfrak{x}_{2 m_{q}+1}\right]_{\propto}\right)\right) \sigma\left(\left[K_{3} \mathfrak{x}_{2 n_{q}}\right]_{\propto},\left[K_{4} \mathfrak{x}_{2 m_{q}+1}\right]_{\propto}\right) \\
& +\delta_{2}\left(\sigma\left(\left[K_{3} \mathfrak{x}_{2 n_{q}}\right]_{\propto},\left[K_{4} \mathfrak{x}_{2 m_{q}+1}\right]_{\propto}\right)\right) \frac{\sigma\left(\left[K_{1} \mathfrak{x}_{2 n_{q}}\right]_{\propto},\left[K_{3} \mathfrak{x}_{2 n_{q}}\right]_{\propto}\right) \sigma\left(\left[K_{2} \mathfrak{x}_{2 m_{q}+1}\right]_{\propto},\left[K_{4} \mathfrak{x}_{2 m_{q}+1}\right]_{\propto}\right)}{1+\sigma\left(\mathfrak{x}_{2 n_{q}}, \mathfrak{x}_{2 m_{q}+1}\right)} \\
& +\delta_{3}\left(\sigma\left(\left[K_{3} \mathfrak{x}_{2 n_{q}}\right]_{\propto},\left[K_{4} \mathfrak{x}_{2 m_{q}+1}\right]_{\propto}\right)\right) \frac{\sigma\left(\left[K_{1} \mathfrak{x}_{2 n_{q}}\right]_{\propto},\left[K_{4} \mathfrak{x}_{2 m_{q}+1}\right]_{\propto}\right) \sigma\left(\left[K_{2} \mathfrak{x}_{2 m_{q}+1}\right]_{\propto},\left[K_{3} \mathfrak{x}_{2 n_{q}}\right]_{\propto}\right)}{1+\sigma\left(\mathfrak{x}_{2 n_{q}}, \mathfrak{x}_{2 m_{q}+1}\right)} \\
& \in s\left(\mathfrak{y}_{2 n_{q}+1},\left[K_{2} \mathfrak{x}_{2 m_{q}+1}\right]_{\propto}\right) .
\end{aligned}
$$

Since $\left[K_{2} \mathfrak{x}_{2 m_{q}+1}\right]_{\propto}$ is a nonempty bounded and closed subset, one can write

$$
\begin{aligned}
& \delta_{1}\left(\sigma\left(\left[K_{3} \mathfrak{x}_{2 n_{q}}\right]_{\propto},\left[K_{4} \mathfrak{x}_{2 m_{q}+1}\right]_{\propto}\right)\right) \sigma\left(\left[K_{3} \mathfrak{x}_{2 n_{q}}\right]_{\propto},\left[K_{4} \mathfrak{x}_{2 m_{q}+1}\right]_{\propto}\right) \\
& +\delta_{2}\left(\sigma\left(\left[K_{3} \mathfrak{x}_{2 n_{q}}\right]_{\propto},\left[K_{4} \mathfrak{x}_{2 m_{q}+1}\right]_{\propto}\right)\right) \frac{\sigma\left(\left[K_{1} \mathfrak{x}_{2 n_{q}}\right]_{\propto},\left[K_{3} \mathfrak{x}_{2 n_{q}}\right]_{\propto}\right) \sigma\left(\left[K_{2} \mathfrak{x}_{2 m_{q}+1}\right]_{\propto},\left[K_{4} \mathfrak{x}_{2 m_{q}+1}\right]_{\propto}\right)}{1+\sigma\left(\mathfrak{x}_{2 n_{q}}, \mathfrak{x}_{2 m_{q}+1}\right)} \\
& +\delta_{3}\left(\sigma\left(\left[K_{3} \mathfrak{x}_{2 n_{q}}\right]_{\propto},\left[K_{4} \mathfrak{x}_{2 m_{q}+1}\right]_{\propto}\right)\right) \frac{\sigma\left(\left[K_{1} \mathfrak{x}_{2 n_{q}}\right]_{\propto},\left[K_{4} \mathfrak{x}_{2 m_{q}+1}\right]_{\propto}\right) \sigma\left(\left[K_{2} \mathfrak{x}_{2 m_{q}+1}\right]_{\propto},\left[K_{3} \mathfrak{x}_{2 n_{q}}\right]_{\propto}\right)}{1+\sigma\left(\mathfrak{x}_{2 n_{q}}, \mathfrak{x}_{2 m_{q}+1}\right)} \\
& \in s\left(\sigma\left(\mathfrak{y}_{2 n_{q}+1}, \mathfrak{y}_{2 m_{q}+2}\right)\right),
\end{aligned}
$$


that is,

$$
\begin{aligned}
\sigma\left(\mathfrak{y}_{2 n_{q}+1}, \mathfrak{y}_{2 m_{q}+2}\right) \preceq & \delta_{1}\left(\sigma\left(\left[K_{3} \mathfrak{x}_{2 n_{q}}\right]_{\propto},\left[K_{4} \mathfrak{x}_{2 m_{q}+1}\right]_{\propto}\right)\right) \sigma\left(\left[K_{3} \mathfrak{x}_{2 n_{q}}\right]_{\propto},\left[K_{4} \mathfrak{x}_{2 m_{q}+1}\right]_{\propto}\right) \\
+ & \delta_{2}\left(\sigma\left(\left[K_{3} \mathfrak{x}_{2 n_{q}}\right]_{\propto},\left[K_{4} \mathfrak{x}_{2 m_{q}+1}\right]_{\propto}\right)\right) \\
& \times \frac{\sigma\left(\left[K_{1} \mathfrak{x}_{2 n_{q}}\right]_{\propto},\left[K_{3} \mathfrak{x}_{2 n_{q}}\right]_{\propto}\right) \sigma\left(\left[K_{2} \mathfrak{x}_{2 m_{q}+1}\right]_{\propto},\left[K_{4} \mathfrak{x}_{2 m_{q}+1}\right]_{\propto}\right)}{1+\sigma\left(\mathfrak{x}_{2 n_{q}}, \mathfrak{x}_{2 m_{q}+1}\right)} \\
+ & \delta_{3}\left(\sigma\left(\left[K_{3} \mathfrak{x}_{2 n_{q}}\right]_{\propto},\left[K_{4} \mathfrak{x}_{2 m_{q}+1}\right]_{\propto}\right)\right) \\
& \times \frac{\sigma\left(\left[K_{1} \mathfrak{x}_{2 n_{q}}\right]_{\propto},\left[K_{4} \mathfrak{x}_{2 m_{q}+1}\right]_{\propto}\right) \sigma\left(\left[K_{2} \mathfrak{x}_{2 m_{q}+1}\right]_{\propto},\left[K_{3} \mathfrak{x}_{2 n_{q}}\right]_{\propto}\right)}{1+\sigma\left(\mathfrak{x}_{2 n_{q}}, \mathfrak{x}_{2 m_{q}+1}\right)} .
\end{aligned}
$$

Utilizing glb property, we have

$$
\begin{aligned}
\sigma\left(\mathfrak{y}_{2 n_{q}+1}, \mathfrak{y}_{2 m_{q}+2}\right) \preceq & \delta_{1}\left(\sigma\left(\mathfrak{y}_{2 n_{q}}, \mathfrak{y}_{2 m_{q}+1}\right)\right) \sigma\left(\mathfrak{y}_{2 n_{q}}, \mathfrak{y}_{2 m_{q}+1}\right) \\
& +\delta_{2}\left(\sigma\left(\mathfrak{y}_{2 n_{q}}, \mathfrak{y}_{2 m_{q}+1}\right)\right) \frac{\sigma\left(\mathfrak{y}_{2 n_{q}+1}, \mathfrak{y}_{2 n_{q}}\right) \sigma\left(\mathfrak{y}_{2 m_{q}+2}, \mathfrak{y}_{2 m_{q}+1}\right)}{1+\sigma\left(\mathfrak{y}_{2 n_{q}}, \mathfrak{y}_{2 m_{q}+1}\right)} \\
& +\delta_{3}\left(\sigma\left(\mathfrak{y}_{2 n_{q}}, \mathfrak{y}_{2 m_{q}+1}\right)\right) \frac{\sigma\left(\mathfrak{y}_{2 n_{q}+1}, \mathfrak{y}_{2 m_{q}+1}\right) \sigma\left(\mathfrak{y}_{2 m_{q}+2}, \mathfrak{y}_{2 n_{q}}\right)}{1+\sigma\left(\mathfrak{y}_{2 n_{q}}, \mathfrak{y}_{2 m_{q}+1}\right)} .
\end{aligned}
$$

This implies that

$$
\begin{aligned}
& \left|\sigma\left(\mathfrak{y}_{2 n_{q}+1}, \mathfrak{y}_{2 m_{q}+2}\right)\right| \leq \delta_{1}\left(\sigma\left(\mathfrak{y}_{2 n_{q}}, \mathfrak{y}_{2 m_{q}+1}\right)\right)\left|\sigma\left(\mathfrak{y}_{2 n_{q}}, \mathfrak{y}_{2 m_{q}+1}\right)\right| \\
& +\delta_{2}\left(\sigma\left(\mathfrak{y}_{2 n_{q}}, \mathfrak{y}_{2 m_{q}+1}\right)\right) \frac{\left|\sigma\left(\mathfrak{y}_{2 n_{q}+1}, \mathfrak{y}_{2 n_{q}}\right)\right|\left|\sigma\left(\mathfrak{y}_{2 m_{q}+2}, \mathfrak{y}_{2 m_{q}+1}\right)\right|}{1+\left|\sigma\left(\mathfrak{y}_{2 n_{q}}, \mathfrak{y}_{2 m_{q}+1}\right)\right|} \\
& +\delta_{3}\left(\sigma\left(\mathfrak{y}_{2 n_{q}}, \mathfrak{y}_{2 m_{q}+1}\right)\right) \\
& \quad \times \frac{\left(\left|\sigma\left(\mathfrak{y}_{2 n_{q}+1}, \mathfrak{y}_{2 n_{q}}\right)\right|+\left|\sigma\left(\mathfrak{y}_{2 n_{q}}, \mathfrak{y}_{2 m_{q}+1}\right)\right|\right)\left(\left|\sigma\left(\mathfrak{y}_{2 m_{q}+2}, \mathfrak{y}_{2 m_{q}+1}\right)\right|+\left|\sigma\left(\mathfrak{y}_{2 m_{q}+1}, \mathfrak{y}_{2 n_{q}}\right)\right|\right)}{1+\left|\sigma\left(\mathfrak{y}_{2 n_{q}}, \mathfrak{y}_{2 m_{q}+1}\right)\right|} .
\end{aligned}
$$

Now, (3.8) yields

$$
\begin{array}{r}
\left|\sigma\left(\mathfrak{y}_{2 n_{q}}, \mathfrak{y}_{2 m_{q}+1}\right)\right| \leq\left|\sigma\left(\mathfrak{y}_{2 n_{q}}, \mathfrak{y}_{2 n_{q}+1}\right)\right|+\left|\sigma\left(\mathfrak{y}_{2 n_{q}+1}, \mathfrak{y}_{2 m_{q}+2}\right)\right|+\left|\sigma\left(\mathfrak{y}_{2 m_{q}+2}, \mathfrak{y}_{2 m_{q}+1}\right)\right| \\
\leq\left|\sigma\left(\mathfrak{y}_{2 n_{q}}, \mathfrak{y}_{2 n_{q}+1}\right)\right|+\delta_{1}\left(\sigma\left(\mathfrak{y}_{2 n_{q}}, \mathfrak{y}_{2 m_{q}+1}\right)\right)\left|\sigma\left(\mathfrak{y}_{2 n_{q}}, \mathfrak{y}_{2 m_{q}+1}\right)\right| \\
\quad+\delta_{2}\left(\sigma\left(\mathfrak{y}_{2 n_{q}}, \mathfrak{y}_{2 m_{q}+1}\right)\right) \frac{\left|\sigma\left(\mathfrak{y}_{2 n_{q}+1}, \mathfrak{y}_{2 n_{q}}\right)\right|\left|\sigma\left(\mathfrak{y}_{2 m_{q}+2}, \mathfrak{y}_{2 m_{q}+1}\right)\right|}{1+\left|\sigma\left(\mathfrak{y}_{2 n_{q}}, \mathfrak{y}_{2 m_{q}+1}\right)\right|} \\
+\delta_{3}\left(\sigma\left(\mathfrak{y}_{2 n_{q}}, \mathfrak{y}_{2 m_{q}+1}\right)\right) \\
\times \frac{\left(\left|\sigma\left(\mathfrak{y}_{2 n_{q}+1}, \mathfrak{y}_{2 n_{q}}\right)\right|+\left|\sigma\left(\mathfrak{y}_{2 n_{q}}, \mathfrak{y}_{2 m_{q}+1}\right)\right|\right)\left(\left|\sigma\left(\mathfrak{y}_{2 m_{q}+2}, \mathfrak{y}_{2 m_{q}+1}\right)\right|+\left|\sigma\left(\mathfrak{y}_{2 m_{q}+1}, \mathfrak{y}_{2 n_{q}}\right)\right|\right)}{1+\left|\sigma\left(\mathfrak{y}_{2 n_{q}}, \mathfrak{y}_{2 m_{q}+1}\right)\right|}+\left|\sigma\left(\mathfrak{y}_{2 m_{q}+2}, \mathfrak{y}_{2 m_{q}+1}\right)\right| .
\end{array}
$$

Taking $q \rightarrow \infty$ and using (3.3) and (3.7), we conclude that

$$
\begin{aligned}
|\epsilon| & \leq \lim _{q \rightarrow \infty}\left(\delta_{1}+\delta_{3}\right)\left(\sigma\left(\mathfrak{y}_{2 n_{q}}, \mathfrak{y}_{2 m_{q}+1}\right)\right)|\epsilon| \\
& \leq \lim _{q \rightarrow \infty}\left(\delta_{1}+\delta_{3}\right)\left(\sigma\left(\mathfrak{y}_{2 n_{q}}, \mathfrak{y}_{2 m_{q}+1}\right)\right)|\epsilon| \leq|\epsilon| \Rightarrow \lim _{q \rightarrow \infty}\left(\delta_{1}+\delta_{3}\right)\left(\sigma\left(\mathfrak{y}_{2 n_{q}}, \mathfrak{y}_{2 m_{q}+1}\right)\right)=1 .
\end{aligned}
$$

Since $\delta_{1}+\delta_{3}: \complement_{+} \rightarrow[0,1)$, we get that $\left|\sigma\left(\mathfrak{y}_{2 n_{q}}, \mathfrak{y}_{2 m_{q}+1}\right)\right| \rightarrow 0$ as $q \rightarrow \infty$, which is a contradiction. Hence, $\left\{\mathfrak{y}_{2 n_{q}}\right\}$ is a C-Cauchy sequence. Consequently, $\left\{\mathfrak{y}_{n}\right\}$ is a C-Cauchy sequence. But $\mathcal{S}$ is C-Complete, so there exists a point $\ddot{t} \in \mathcal{S}$ with $\mathfrak{y}_{n} \rightarrow \ddot{t}$ as $n \rightarrow \infty$. Moreover, we have to show that $\ddot{t} \in\left[K_{1} \ddot{t}\right]_{\propto}$ and $\ddot{t} \in\left[K_{2} \ddot{t}\right]_{\propto}$ for all $\ddot{t} \in \mathcal{N}$. For this we 
consider (3.1) with $\mathfrak{x}=\mathfrak{y}_{2 n}$ and $\mathfrak{y}=\ddot{t}$, that is,

$$
\begin{aligned}
& \delta_{1}\left(\sigma\left(\left[K_{3} \mathfrak{y}_{2 n}\right]_{\propto},\left[K_{4} \ddot{t}\right]_{\propto}\right)\right) \sigma\left(\left[K_{3} \mathfrak{y}_{2 n}\right]_{\propto},\left[K_{4} \ddot{t}\right]_{\propto}\right) \\
& +\delta_{2}\left(\sigma\left(\left[K_{3} \mathfrak{y}_{2 n}\right]_{\propto},\left[K_{4} \ddot{t}\right]_{\propto}\right)\right) \frac{\sigma\left(\left[K_{1} \mathfrak{y}_{2 n}\right]_{\propto},\left[K_{3} \mathfrak{y}_{2 n}\right]_{\propto}\right) \sigma\left(\left[K_{2} \ddot{t}\right]_{\propto,},\left[K_{4} \ddot{t}\right]_{\propto}\right)}{1+\sigma\left(\mathfrak{y}_{2 n}, \ddot{t}\right)} \\
& +\delta_{3}\left(\sigma\left(\left[K_{3} \mathfrak{y}_{2 n}\right]_{\propto},\left[K_{4} \ddot{t}\right]_{\propto}\right)\right) \frac{\sigma\left(\left[K_{1} \mathfrak{y}_{2 n}\right]_{\propto},\left[K_{4} \ddot{t}\right]_{\propto}\right) \sigma\left(\left[K_{2} \ddot{t}\right]_{\propto},\left[K_{3} \mathfrak{y}_{2 n}\right]_{\propto}\right)}{1+\sigma\left(\mathfrak{y}_{2 n}, \ddot{t}\right)} \\
& \in s\left(\left[K_{1} \mathfrak{y}_{2 n}\right]_{\propto},\left[K_{2} \ddot{t}\right]_{\propto}\right) .
\end{aligned}
$$

Since $\mathfrak{y}_{2 n+1} \in\left[K_{1} \mathfrak{y}_{2 n}\right]_{\propto}$, we get

$$
\begin{aligned}
& \delta_{1}\left(\sigma\left(\left[K_{3} \mathfrak{y}_{2 n}\right]_{\propto},\left[K_{4} \ddot{t}\right]_{\propto}\right)\right) \sigma\left(\left[K_{3} \mathfrak{y}_{2 n}\right]_{\propto},\left[K_{4} \ddot{t}\right]_{\propto}\right) \\
& +\delta_{2}\left(\sigma\left(\left[K_{3} \mathfrak{y}_{2 n}\right]_{\propto},\left[K_{4} \ddot{t}\right]_{\propto}\right)\right) \frac{\sigma\left(\left[K_{1} \mathfrak{y}_{2 n}\right]_{\propto},\left[K_{3} \mathfrak{y}_{2 n}\right]_{\propto}\right) \sigma\left(\left[K_{2} \ddot{t}\right]_{\propto},\left[K_{4} \ddot{t}\right]_{\propto}\right)}{1+\sigma\left(\mathfrak{y}_{2 n}, \ddot{t}\right)} \\
& +\delta_{3}\left(\sigma\left(\left[K_{3} \mathfrak{y}_{2 n}\right]_{\propto},\left[K_{4} \ddot{t}\right]_{\propto}\right)\right) \frac{\sigma\left(\left[K_{1} \mathfrak{y}_{2 n}\right]_{\propto},\left[K_{4} \ddot{t}\right]_{\propto}\right) \sigma\left(\left[K_{2} \ddot{t}\right]_{\propto},\left[K_{3} \mathfrak{y}_{2 n}\right]_{\propto}\right)}{1+\sigma\left(\mathfrak{y}_{2 n}, \ddot{t}\right)} \\
& \in s\left(\mathfrak{y}_{2 n+1},\left[K_{2} \ddot{t}\right]_{\propto}\right) .
\end{aligned}
$$

By virtue of the fact that $\left[K_{2} \ddot{t}\right]_{\propto}$ is a nonempty subset, there exists an $r_{n} \in\left[K_{2} \ddot{t}\right]_{\propto}$ such that

$$
\begin{aligned}
& \delta_{1}\left(\sigma\left(\left[K_{3} \mathfrak{y}_{2 n}\right]_{\propto},\left[K_{4} \ddot{t}\right]_{\propto}\right)\right) \sigma\left(\left[K_{3} \mathfrak{y}_{2 n}\right]_{\propto},\left[K_{4} \ddot{t}\right]_{\propto}\right) \\
& +\delta_{2}\left(\sigma\left(\left[K_{3} \mathfrak{y}_{2 n}\right]_{\propto},\left[K_{4} \ddot{t}\right]_{\propto}\right)\right) \frac{\sigma\left(\left[K_{1} \mathfrak{y}_{2 n}\right]_{\propto},\left[K_{3} \mathfrak{y}_{2 n}\right]_{\propto}\right) \sigma\left(\left[K_{2} \ddot{t}\right]_{\propto},\left[K_{4} \ddot{t}\right]_{\propto}\right)}{1+\sigma\left(\mathfrak{y}_{2 n}, \ddot{t}\right)} \\
& +\delta_{3}\left(\sigma\left(\left[K_{3} \mathfrak{y}_{2 n}\right]_{\propto},\left[K_{4} \ddot{t}\right]_{\propto}\right)\right) \frac{\sigma\left(\left[K_{1} \mathfrak{y}_{2 n}\right]_{\propto},\left[K_{4} \ddot{t}\right]_{\propto}\right) \sigma\left(\left[K_{2} \ddot{t}\right]_{\propto},\left[K_{3} \mathfrak{y}_{2 n}\right]_{\propto}\right)}{1+\sigma\left(\mathfrak{y}_{2 n}, \ddot{t}\right)} \\
& \in s\left(\sigma\left(\mathfrak{y}_{2 n+1}, r_{n}\right)\right),
\end{aligned}
$$

that is,

$$
\begin{aligned}
\sigma\left(\mathfrak{y}_{2 n+1}, r_{n}\right) \preceq & \delta_{1}\left(\sigma\left(\left[K_{3} \mathfrak{y}_{2 n}\right]_{\propto},\left[K_{4} \ddot{t}\right]_{\propto}\right)\right) \sigma\left(\left[K_{3} \mathfrak{y}_{2 n}\right]_{\propto},\left[K_{4} \ddot{t}\right]_{\propto}\right) \\
& +\delta_{2}\left(\sigma\left(\left[K_{3} \mathfrak{y}_{2 n}\right]_{\propto},\left[K_{4} \ddot{t}\right]_{\propto}\right)\right) \frac{\sigma\left(\left[K_{1} \mathfrak{y}_{2 n}\right]_{\propto},\left[K_{3} \mathfrak{y}_{2 n}\right]_{\propto}\right) \sigma\left(\left[K_{2} \ddot{t}\right]_{\propto},\left[K_{4} \ddot{t}\right]_{\propto}\right)}{1+\sigma\left(\mathfrak{y}_{2 n}, \ddot{t}\right)} \\
& +\delta_{3}\left(\sigma\left(\left[K_{3} \mathfrak{y}_{2 n}\right]_{\propto},\left[K_{4} \ddot{t}\right]_{\propto}\right)\right) \frac{\sigma\left(\left[K_{1} \mathfrak{y}_{2 n}\right]_{\propto},\left[K_{4} \ddot{t}\right]_{\propto}\right) \sigma\left(\left[K_{2} \ddot{t}\right]_{\propto},\left[K_{3} \mathfrak{y}_{2 n}\right]_{\propto}\right)}{1+\sigma\left(\mathfrak{y}_{2 n}, \ddot{t}\right)} .
\end{aligned}
$$

Applying glb property, we have

$$
\begin{aligned}
\sigma\left(\mathfrak{y}_{2 n+1}, r_{n}\right) \preceq & \delta_{1}\left(\sigma\left(\mathfrak{y}_{2 n}, \ddot{t}\right)\right) \sigma\left(\mathfrak{y}_{2 n}, \ddot{t}\right)+\delta_{2}\left(\sigma\left(\mathfrak{y}_{2 n}, \ddot{t}\right)\right) \frac{\sigma\left(\mathfrak{y}_{2 n+1}, \mathfrak{y}_{2 n}\right) \sigma\left(r_{n}, \tau\right)}{1+\sigma\left(\mathfrak{y}_{2 n}, \ddot{t}\right)} \\
& +\delta_{3}\left(\sigma\left(\mathfrak{y}_{2 n}, \ddot{t}\right)\right) \frac{\sigma\left(\mathfrak{y}_{2 n+1}, \ddot{t}\right) \sigma\left(r_{n}, \mathfrak{y}_{2 n}\right)}{1+\sigma\left(\mathfrak{y}_{2 n}, \ddot{t}\right)} .
\end{aligned}
$$

Using triangular inequality, we obtain

$$
\begin{aligned}
\sigma\left(\ddot{t}, r_{n}\right) \preceq & \sigma\left(\ddot{t}, \mathfrak{y}_{2 n+1}\right)+\sigma\left(\mathfrak{y}_{2 n+1}, r_{n}\right) \\
\preceq & \sigma\left(\ddot{t}, \mathfrak{y}_{2 n+1}\right)+\delta_{1}\left(\sigma\left(\mathfrak{y}_{2 n}, \ddot{t}\right)\right) \sigma\left(\mathfrak{y}_{2 n}, \ddot{t}\right)+\delta_{2}\left(\sigma\left(\mathfrak{y}_{2 n}, \ddot{t}\right)\right) \frac{\sigma\left(\mathfrak{y}_{2 n+1}, \mathfrak{y}_{2 n}\right) \sigma\left(r_{n}, \tau\right)}{1+\sigma\left(\mathfrak{y}_{2 n}, \ddot{t}\right)} \\
& +\delta_{3}\left(\sigma\left(\mathfrak{y}_{2 n}, \ddot{t}\right)\right) \frac{\sigma\left(\mathfrak{y}_{2 n+1}, \ddot{t}\right) \sigma\left(r_{n}, \mathfrak{y}_{2 n}\right)}{1+\sigma\left(\mathfrak{y}_{2 n}, \ddot{t}\right)} .
\end{aligned}
$$


Furthermore, we deduce that

$$
\begin{aligned}
\left|\sigma\left(\ddot{t}, r_{n}\right)\right| \leq & \left|\sigma\left(\ddot{t}, \mathfrak{y}_{2 n+1}\right)\right|+\delta_{1}\left(\sigma\left(\mathfrak{y}_{2 n}, \ddot{t}\right)\right)\left|\sigma\left(\mathfrak{y}_{2 n}, \ddot{t}\right)\right|+\delta_{2}\left(\sigma\left(\mathfrak{y}_{2 n}, \ddot{t}\right)\right) \frac{\left|\sigma\left(\mathfrak{y}_{2 n+1}, \mathfrak{y}_{2 n}\right)\right|\left|\sigma\left(r_{n}, \tau\right)\right|}{1+\left|\sigma\left(\mathfrak{y}_{2 n}, \ddot{t}\right)\right|} \\
& +\delta_{3}\left(\sigma\left(\mathfrak{y}_{2 n}, \ddot{t}\right)\right) \frac{\left|\sigma\left(\mathfrak{y}_{2 n+1}, \ddot{t} \mid\right)\right| \sigma\left(r_{n}, \mathfrak{y}_{2 n}\right) \mid}{1+\left|\sigma\left(\mathfrak{y}_{2 n}, \ddot{t}\right)\right|} .
\end{aligned}
$$

If we take limit $n \rightarrow \infty$, then the latter inequality yields that $\left|\sigma\left(\ddot{t}, r_{n}\right)\right| \rightarrow 0$, and thus we get $r_{n} \rightarrow \ddot{t}$ as $n \rightarrow \infty$. Since we have supposed that $\left[K_{2} \ddot{t}\right]_{\propto}$ is a nonempty bounded and closed subset of $\mathcal{S}$, so $\ddot{t} \in\left[K_{2} \ddot{t}\right]_{\propto}$. Moreover, we derive from the construction of sequence that $\ddot{t} \in\left[K_{2} \ddot{t}\right]_{\propto} \subseteq\left[K_{3} \ddot{t}\right]_{\propto}$. Similarly, we can obtain $\ddot{t} \in\left[K_{1} \ddot{t}\right]_{\propto} \subseteq\left[K_{4} \ddot{t}\right]_{\propto}$. Thus, $\ddot{t}$ is a common fuzzy fixed point of $K_{1}, K_{2}, K_{3}, K_{4}$.

Corollary 3.2. Let $(\mathcal{S}, \sigma)$ be a $C$-complete complex valued metric space, and $K_{1}, K_{2}, K_{3}, K_{4}$ : $\mathcal{S} \rightarrow \mathfrak{S}(\mathcal{S})$ are fuzzy mappings satisfying the glb property with each $\mathfrak{x} \in \mathcal{S}$ and for $\propto \in(0,1]$ there exist $\left[K_{1} \mathfrak{x}\right]_{\propto},\left[K_{2} \mathfrak{x}\right]_{\propto},\left[K_{3} \mathfrak{x}\right]_{\propto},\left[K_{4} \mathfrak{x}\right]_{\propto}$ (nonempty closed bounded subsets of $\mathcal{S}$ ). Suppose the following assumption holds:

$$
\begin{aligned}
& \delta_{1} \sigma\left(\left[K_{3} \mathfrak{x}\right]_{\propto},\left[K_{4} \mathfrak{y}\right]_{\propto}\right)+\delta_{2} \frac{\sigma\left(\left[K_{1} \mathfrak{x}\right]_{\propto},\left[K_{3} \mathfrak{x}\right]_{\propto}\right) \sigma\left(\left[K_{2} \mathfrak{y}\right]_{\propto},\left[K_{4} \mathfrak{y}\right]_{\propto}\right)}{1+\sigma(\mathfrak{x}, \mathfrak{y})} \\
& +\delta_{3} \frac{\sigma\left(\left[K_{1} \mathfrak{x}\right]_{\propto},\left[K_{4} \mathfrak{y}\right]_{\propto}\right) \sigma\left(\left[K_{2} \mathfrak{y}\right]_{\propto},\left[K_{3} \mathfrak{x}\right]_{\propto}\right)}{1+\sigma(\mathfrak{x}, \mathfrak{y})} \\
& \in s\left(\left[K_{1} \mathfrak{x}\right]_{\propto},\left[K_{2} \mathfrak{y}\right]_{\propto}\right)
\end{aligned}
$$

for each $\mathfrak{y}, \mathfrak{x} \in \mathcal{S}$, and for each $\delta_{i} \in \mathfrak{R}_{+}, i=1,2,3$, where $\delta_{1}+\delta_{2}+\delta_{3}<1$. If $\left[K_{1}(\mathcal{S})\right]_{\propto},\left[K_{2}(\mathcal{S})\right]_{\propto}$, $\left[K_{3}(\mathcal{S})\right]_{\propto},\left[K_{4}(\mathcal{S})\right]_{\propto}$ are closed sets and $\left[K_{1}(\mathcal{S})\right]_{\propto} \subseteq\left[K_{4}(\mathcal{S})\right]_{\propto}$ and $\left[K_{2}(\mathcal{S})\right]_{\propto} \subseteq\left[K_{3}(\mathcal{S})\right]_{\propto}$, then there exists a common fuzzy fixed point of $K_{1}, K_{2}, K_{3}, K_{4}$ in $\mathcal{S}$.

Corollary 3.3. Assume that $(\mathcal{S}, \sigma)$ is a C-complete complex valued metric space and $K_{1}, K_{2}: \mathcal{S} \rightarrow \mathfrak{S}(\mathcal{S})$ are fuzzy mappings satisfying the glb property. Let $\delta_{1}, \delta_{2}, \delta_{3}: \complement_{+} \rightarrow$ $[0,1)$ be given mappings such that for each $\mathfrak{x} \in \mathcal{S}$ and $\propto \in(0,1]$ there exist $\left[K_{1} \mathfrak{x}\right]_{\propto},\left[K_{2} \mathfrak{x}\right]_{\propto}$ (nonempty bounded and closed subsets of $\mathcal{S})$. Suppose that assumptions given below hold:

i) $\sum_{w=1}^{3} \delta_{w}(\mathfrak{x})<1$ and the mapping $£: \complement_{+} \rightarrow[0,1)$ is defined by

$$
£(\mathfrak{x})=\frac{\delta_{1}(\mathfrak{x})}{1-\delta_{2}(\mathfrak{x})}
$$

for all $\mathfrak{x} \in \complement_{+}, £ \in \Gamma$;

ii) for each $\mathfrak{y}, \mathfrak{x} \in \mathcal{S}$, we have

$$
\begin{aligned}
& \delta_{1}(\sigma(\mathfrak{x}, \mathfrak{y})) \sigma(\mathfrak{x}, \mathfrak{y})+\delta_{2}(\sigma(\mathfrak{x}, \mathfrak{y})) \frac{\sigma\left(\left[K_{1} \mathfrak{x}\right]_{\propto}, \mathfrak{x}\right) \sigma\left(\left[K_{2} \mathfrak{y}\right]_{\propto}, \mathfrak{y}\right)}{1+\sigma(\mathfrak{x}, \mathfrak{y})} \\
& +\delta_{3}(\sigma(\mathfrak{x}, \mathfrak{y})) \frac{\sigma\left(\left[K_{1} \mathfrak{x}\right]_{\propto}, \mathfrak{y}\right) \sigma\left(\left[K_{2} \mathfrak{y}\right]_{\propto}, \mathfrak{x}\right)}{1+\sigma(\mathfrak{x}, \mathfrak{y})} \in s\left(\left[K_{1} \mathfrak{x}\right]_{\propto},\left[K_{2} \mathfrak{y}\right]_{\propto}\right) .
\end{aligned}
$$

Then there exists a common fuzzy fixed point of $K_{1}, K_{2}$ in $\mathcal{S}$.

Corollary 3.4. Conclusion of Corollary 3.3 remains intact if condition ii) is replaced by condition

$$
\delta_{1}(\sigma(\mathfrak{x}, \mathfrak{y})) \sigma(\mathfrak{x}, \mathfrak{y})+\delta_{2}(\sigma(\mathfrak{x}, \mathfrak{y})) \frac{\sigma\left(\left[K_{1} \mathfrak{x}\right]_{\propto}, \mathfrak{y}\right) \sigma\left(\left[K_{2} \mathfrak{y}\right]_{\propto}, \mathfrak{x}\right)}{1+\sigma(\mathfrak{x}, \mathfrak{y})} \in s\left(\left[K_{1} \mathfrak{x}\right]_{\propto},\left[K_{2} \mathfrak{y}\right]_{\propto}\right) .
$$

Corollary 3.5. Assume that $(\mathcal{S}, \sigma)$ is a $C$-complete complex valued metric space and $K_{1}$ : $\mathcal{S} \rightarrow \mathfrak{S}(\mathcal{S})$ is a fuzzy mapping satisfying the glb property. Let $\delta_{1}, \delta_{2}, \delta_{3}: \complement_{+} \rightarrow[0,1)$ be given mappings such that for each $\mathfrak{x} \in \mathcal{S}$ and $\propto \in(0,1]$ there exists a nonempty bounded and closed subset $\left[K_{1} \mathfrak{x}\right]_{\propto}$ of $\mathcal{S}$. Suppose further that the following conditions hold: 
i) $\sum_{w=1}^{3} \delta_{w}(\mathfrak{x})<1$ and the mapping $£: \complement_{+} \rightarrow[0,1)$ is defined by

$$
£(\mathfrak{x})=\frac{\delta_{1}(\mathfrak{x})}{1-\delta_{2}(\mathfrak{x})}
$$

for all $\mathfrak{x} \in \complement_{+}, £ \in \Gamma$;

ii) for every $\mathfrak{y}, \mathfrak{x} \in \mathcal{S}$,

$$
\begin{aligned}
& \delta_{1}(\sigma(\mathfrak{x}, \mathfrak{y})) \sigma(\mathfrak{x}, \mathfrak{y})+\delta_{2}(\sigma(\mathfrak{x}, \mathfrak{y})) \frac{\sigma\left(\left[K_{1} \mathfrak{x}\right]_{\propto}, \mathfrak{x}\right) \sigma\left(\left[K_{1} \mathfrak{y}\right]_{\propto}, \mathfrak{y}\right)}{1+\sigma(\mathfrak{x}, \mathfrak{y})} \\
& +\delta_{3}(\sigma(\mathfrak{x}, \mathfrak{y})) \frac{\sigma\left(\left[K_{1} \mathfrak{x}\right]_{\propto}, \mathfrak{y}\right) \sigma\left(\left[K_{1} \mathfrak{y}\right]_{\propto}, \mathfrak{x}\right)}{1+\sigma(\mathfrak{x}, \mathfrak{y})} \in s\left(\left[K_{1} \mathfrak{x}\right]_{\propto},\left[K_{1} \mathfrak{y}\right]_{\propto}\right) .
\end{aligned}
$$

Then $K_{1}$ has a fuzzy fixed point in $\mathcal{S}$.

\section{Applications}

Theorem 4.1. Let $(\mathcal{S}, \sigma)$ be a complex valued metric space and $\Psi_{1}, \Psi_{2}, \Psi_{3}, \Psi_{4}: \mathcal{S} \rightarrow$ $C B(\mathcal{S})$ be multivalued mappings. Assume that $\delta_{l}(\mathfrak{x}): \complement \rightarrow[0,1), l=1,2,3$,

i) $\delta_{1}(\mathfrak{x})+\delta_{2}(\mathfrak{x})+\delta_{3}(\mathfrak{x})<1$ and the mapping $£: \complement_{+} \rightarrow[0,1)$ is defined by

$$
£(\mathfrak{x})=\frac{\delta_{1}(\mathfrak{x})}{1-\delta_{2}(\mathfrak{x})}
$$

for all $\mathfrak{x} \in \complement_{+}, £ \in \Gamma$;

ii) for each $\mathfrak{x}, \mathfrak{y} \in \mathcal{S}$,

$$
\begin{aligned}
& \delta_{1}\left(\sigma\left(\Psi_{3} \mathfrak{x}, \Psi_{4} \mathfrak{y}\right)\right) \sigma\left(\Psi_{3} \mathfrak{x}, \Psi_{4} \mathfrak{y}\right)+\delta_{2}\left(\sigma\left(\Psi_{3} \mathfrak{x}, \Psi_{4} \mathfrak{y}\right)\right) \frac{\sigma\left(\Psi_{1} \mathfrak{x}, \Psi_{3} \mathfrak{x}\right) \sigma\left(\Psi_{2} \mathfrak{y}, \Psi_{4} \mathfrak{y}\right)}{1+\sigma(\mathfrak{x}, \mathfrak{y})} \\
& +\delta_{3}\left(\sigma\left(\Psi_{3} \mathfrak{x}, \Psi_{4} \mathfrak{y}\right)\right) \frac{\sigma\left(\Psi_{1} \mathfrak{x}, \Psi_{4} \mathfrak{y}\right) \sigma\left(\Psi_{2} \mathfrak{y}, \Psi_{3} \mathfrak{x}\right)}{1+\sigma(\mathfrak{x}, \mathfrak{y})} \in s\left(\Psi_{1} \mathfrak{x}, \Psi_{2} \mathfrak{y}\right) .
\end{aligned}
$$

If $\Psi_{1}(\mathcal{S}), \Psi_{2}(\mathcal{S}), \Psi_{3}(\mathcal{S}), \Psi_{4}(\mathcal{S})$ are closed and $\Psi_{1}(\mathcal{S}) \subseteq \Psi_{4}(\mathcal{S}), \Psi_{2}(\mathcal{S}) \subseteq \Psi_{3}(\mathcal{S})$, then $\Psi_{1}, \Psi_{2}, \Psi_{3}, \Psi_{4}$ have a fuzzy common fixed point in $\mathcal{S}$.

Proof. Let $K_{l}: \mathcal{S} \rightarrow \mathfrak{S}(\mathcal{S}), l=1,2,3,4$ be fuzzy mappings defined as

$$
\begin{aligned}
& K_{1}(\mathfrak{x})= \begin{cases}\propto, & \text { if } \mathfrak{x} \in \Psi_{1} \mathfrak{x}, \\
0, & \text { if } \mathfrak{x} \notin \Psi_{1} \mathfrak{x},\end{cases} \\
& K_{2}(\mathfrak{x})= \begin{cases}\propto, & \text { if } \mathfrak{x} \in \Psi_{2} \mathfrak{x}, \\
0, & \text { if } \mathfrak{x} \notin \Psi_{2} \mathfrak{x},\end{cases} \\
& K_{3}(\mathfrak{x})=\left\{\begin{array}{cc}
\propto, & \text { if } \mathfrak{x} \in \Psi_{3} \mathfrak{x}, \\
0, & \text { if } \mathfrak{x} \notin \Psi_{3} \mathfrak{x},
\end{array}\right. \\
& K_{4}(\mathfrak{x})=\left\{\begin{array}{cc}
\propto, & \text { if } \mathfrak{x} \in \Psi_{4} \mathfrak{x}, \\
0, & \text { if } \mathfrak{x} \notin \Psi_{4} \mathfrak{x}
\end{array}\right.
\end{aligned}
$$

Then for any $\propto \in(0,1],\left[K_{1} \mathfrak{x}\right]_{\propto}=\Psi_{1} \mathfrak{x},\left[K_{2} \mathfrak{x}\right]_{\propto}=\Psi_{2} \mathfrak{x},\left[K_{3} \mathfrak{x}\right]_{\propto}=\Psi_{3} \mathfrak{x}$ and $\left[K_{4} \mathfrak{x}\right]_{\propto}=\Psi_{4} \mathfrak{x}$. Therefore, for every $\mathfrak{x}, \mathfrak{y} \in \mathcal{S}, s\left(\left[L_{1} \mathfrak{x}\right]_{\propto},\left[K_{2} \mathfrak{y}\right]_{\propto}\right)=s\left(\Psi_{1} \mathfrak{x}, \Psi_{2} \mathfrak{y}\right)$, so one can apply Theorem 3.1 to get the common fixed point.

Now we are demonstrating applications of Corollary 3.4 to the existence of the common solution of second-order nonlinear boundary value problems

$$
\left\{\begin{array}{l}
\mathfrak{x}^{\prime \prime}(\jmath)=\mathbb{k}\left(\jmath, \mathfrak{x}(\jmath), \mathfrak{x}^{\prime}(\jmath)\right), \quad \text { if } \jmath \in[0, \Lambda], \quad \Lambda>0, \\
\mathfrak{x}\left(\jmath_{1}\right)=\mathfrak{x}_{1}, \\
\mathfrak{x}\left(\jmath_{2}\right)=\mathfrak{x}_{2}, \quad \jmath_{1}, \jmath_{1} \in[0, \Lambda],
\end{array}\right.
$$


where $\mathbb{k}:[0, \Lambda] \times \mathfrak{S}(\mathcal{S}) \times \mathfrak{S}(\mathcal{S}) \rightarrow \mathfrak{S}(\mathcal{S})$ is a continuous function. This problem is equivalent to the integral equation

$$
\mathfrak{x}(\jmath)=\int_{\jmath_{1}}^{\jmath_{2}} G(\jmath, \wp) \mathbb{k}\left(\wp, \mathfrak{x}(\wp), \mathfrak{x}^{\prime}(\wp)\right) d \wp+\lambda(\jmath), \quad \jmath \in[0, \Lambda]
$$

where the Green function $G$ is given by

$$
G(\jmath, \wp)= \begin{cases}\frac{\left(\jmath_{2}-\jmath\right)\left(\wp-\jmath_{1}\right)}{\jmath_{2}-\jmath_{1}}, & \text { if } \jmath_{1} \leq \wp \leq \jmath \leq \jmath_{2}, \\ \frac{\left(\jmath_{2}-\wp\right)\left(\jmath-\jmath_{1}\right)}{\jmath_{2}-\jmath_{1}}, & \text { if } \jmath_{1} \leq \jmath \leq \wp \leq \jmath_{2},\end{cases}
$$

and $\lambda(\jmath)$ satisfies $\lambda^{\prime \prime}=0, \lambda\left(\jmath_{1}\right)=\mathfrak{x}_{1}, \lambda\left(\jmath_{2}\right)=\mathfrak{x}_{2} \cdot G(\jmath, \wp)$ has the properties

$$
\int_{\jmath_{1}}^{\jmath_{2}} G(\jmath, \wp) d \wp \leq \frac{\left(\jmath_{2}-\jmath_{1}\right)^{2}}{8}
$$

and

$$
\int_{\jmath_{1}}^{\jmath_{2}} G_{\jmath}(\jmath, \wp) d \wp \leq \frac{\jmath_{2}-\jmath_{1}}{2} .
$$

For brief study, we refer the reader to $[22,25,27]$. We are intend to prove our result by obtaining the existence of the common solution of the integral operators defined as

$$
L_{l}(\mathfrak{x})(\jmath)=\int_{\jmath_{1}}^{\jmath_{2}} G(\jmath, \wp) \mathbb{k}_{l}\left(\wp, \mathfrak{x}(\wp), \mathfrak{x}^{\prime}(\wp)\right) d \wp+\lambda(\jmath), \quad \jmath \in[0, \Lambda], \quad l \in\{1,2\},
$$

where $\mathbb{k}_{l} \in C([0, \Lambda] \times \mathfrak{S}(\mathcal{S}) \times \mathfrak{S}(\mathcal{S}), \mathfrak{S}(\mathcal{S})), l=1,2, \mathfrak{x} \in C^{1}([0, \Lambda], \mathfrak{S}(\mathcal{S}))$ and $\lambda \in C([0, \Lambda], \mathfrak{S}(\mathcal{S}))$.

Throughout this section, we mention

$$
\Xi_{l}(\mathfrak{x}(\jmath))=\int_{\jmath 1}^{\jmath_{2}} G(\jmath, \wp) \mathbb{k}_{l}\left(\wp, \mathfrak{x}(\wp), \mathfrak{x}^{\prime}(\wp)\right) d \wp
$$

and

$$
\Xi_{l}^{\prime}(\mathfrak{x}(\jmath))=\int_{\jmath 1}^{\jmath_{2}} G_{\jmath}(\jmath, \wp) \mathbb{k}_{l}\left(\wp, \mathfrak{x}(\wp), \mathfrak{x}^{\prime}(\wp)\right) d \wp .
$$

Theorem 4.2. Consider integral equation (4.2). Suppose that the following hypotheses hold for every $\mathrm{\jmath} \in[0, \Lambda]$ :

$\mathrm{H} 1) \mathbb{k}_{l}:[0, \Lambda] \times \mathfrak{S}(\mathcal{S}) \times \mathfrak{S}(\mathcal{S}) \rightarrow \mathfrak{S}(\mathcal{S}), l=1,2$ are increasing in their second and third variables;

$\mathrm{H} 2)$ there exists some $\mathfrak{x}_{0}(\jmath) \leq \int_{\jmath_{1}}^{\jmath_{2}} G(\jmath, \wp) \mathbb{k}_{i}\left(\wp, \mathfrak{x}(\wp), \mathfrak{x}^{\prime}(\wp)\right) d \wp+\lambda(\jmath)$, where $\jmath_{1}, \jmath_{2} \in[0, \Lambda]$. If there exist mappings $\delta_{1}, \delta_{2}: \complement \rightarrow[0,1)$, which satisfy conditions given below:

i) $\delta_{1}(\mathfrak{x})+\delta_{2}(\mathfrak{x})<1$ and the mapping $£: \complement_{+} \rightarrow[0,1)$ is defined by

$$
£(\mathfrak{x})=\frac{\delta_{1}(\mathfrak{x})}{1-\delta_{2}(\mathfrak{x})}
$$

for all $\mathfrak{x} \in \complement_{+}, £ \in \Gamma$;

ii) for every $\mathfrak{x}, \mathfrak{y} \in \mathcal{S}$ and $\mathfrak{\jmath} \in[0, \Lambda]$,

$$
\begin{aligned}
\left|\mathbb{k}_{1}\left(\jmath, \mathfrak{x}(\jmath), \mathfrak{x}^{\prime}(\jmath)\right)-\mathbb{k}_{2}\left(\jmath, \mathfrak{y}(\jmath), \mathfrak{y}^{\prime}(\jmath)\right)\right| \preceq & \delta_{1}\left(\max _{\jmath \in\left[\jmath_{1}, \jmath_{2}\right]} \mathcal{D}_{\mathfrak{x y}}(\jmath)\right) \mathcal{D}_{\mathfrak{x y}}(\jmath) \\
& +\delta_{2}\left(\max _{\jmath \in\left[\jmath_{1}, \jmath_{2}\right]} \mathcal{D}_{\mathfrak{x y}}(\jmath)\right) \mathcal{G}_{\mathfrak{x y}}(\jmath),
\end{aligned}
$$

where

$$
\mathcal{D}_{\mathfrak{x y}}(\jmath)=\gamma\left|\Xi_{1} \mathfrak{x}(\jmath)-\Xi_{2} \mathfrak{y}(\jmath)\right|+\xi\left|\Xi_{1}^{\prime} \mathfrak{x}(\jmath)-\Xi_{2}^{\prime} \mathfrak{y}(\jmath)\right| \sqrt{1+a^{2}} e^{i \tan a},
$$




$$
\begin{aligned}
\mathcal{G}_{\mathfrak{x} \mathfrak{y}}= & \frac{\gamma\left|\Xi_{1} \mathfrak{x}(\jmath)-\Xi_{2} \mathfrak{y}(\jmath)\right|+\xi\left|\Xi_{1}^{\prime} \mathfrak{x}(\jmath)-\Xi_{2}^{\prime} \mathfrak{y}(\jmath)\right|}{1+\max _{\jmath \in\left[\jmath_{1}, \jmath_{2}\right]} \mathcal{D}_{\mathfrak{x y}}(\jmath)} \\
& \times \gamma\left|\Xi_{2} \mathfrak{y}(\jmath)-\Xi_{1} \mathfrak{x}(\jmath)\right|+\xi\left|\Xi_{2}^{\prime} \mathfrak{y}(\jmath)-\Xi_{1}^{\prime} \mathfrak{x}(\jmath)\right| \sqrt{1+a^{2}} e^{i \tan a}
\end{aligned}
$$

iii) for $\gamma, \xi>0$ and $\jmath_{1}, \jmath_{2} \in[0, \Lambda]$,

$$
\gamma \frac{\left(\jmath_{2}-\jmath_{1}\right)^{2}}{8}+\xi \frac{\left(\jmath_{2}-\jmath_{1}\right)}{2}<1
$$

then the system of nonlinear integral equations

$$
\mathfrak{x}(\jmath)=\int_{\jmath_{1}}^{\jmath_{2}} G(\jmath, \wp) \mathbb{k}_{l}\left(\wp, x(\wp), x^{\prime}(\wp)\right) d \wp+\lambda(\jmath), \quad \jmath \in[0, \Lambda], \quad l \in\{1,2\}
$$

has a common solution in $C^{1}\left(\left[\jmath_{1}, \jmath_{2}\right], \mathfrak{S}(\mathcal{S})\right)$.

Proof. Let $\mathcal{M}=C^{1}\left(\left[\jmath_{1}, \jmath_{2}\right], \mathfrak{S}(\mathcal{S})\right)$ and $d: \mathcal{M} \times \mathcal{M} \rightarrow \complement$ be defined as

$$
d(\mathfrak{y}, \mathfrak{x})=\max _{\jmath \in\left[\jmath_{1}, \jmath_{2}\right]}\left(\gamma|\mathfrak{y}(\jmath)-\mathfrak{x}(\jmath)|+\xi\left|\mathfrak{y}^{\prime}(\jmath)-\mathfrak{x}^{\prime}(\jmath)\right|\right) \sqrt{1+c^{2}} e^{i \tan c} .
$$

Define integral operators $L_{l}: \mathcal{M} \rightarrow \mathcal{M}, l=1,2$ by

$$
L_{l}(\mathfrak{x})(\jmath)=\int_{\jmath_{1}}^{\jmath_{2}} G(\jmath, \wp) \mathbb{k}_{i}\left(\wp, \mathfrak{x}(\wp), \mathfrak{x}^{\prime}(\wp)\right) d \wp+\lambda(\jmath),
$$

where $\mathbb{k}_{i} \in C([0, \Lambda] \times \mathfrak{S}(\mathcal{S}) \times \mathfrak{S}(\mathcal{S}), \mathfrak{S}(\mathcal{S})), \mathfrak{x} \in C^{1}([0, \Lambda], \mathfrak{S}(\mathcal{S}))$, and $\lambda \in C([0, \Lambda], \mathfrak{S}(\mathcal{S}))$. For $\mathfrak{x}, \mathfrak{y} \in \mathcal{S}$, we obtain $\left[L_{l} \mathfrak{x}\right]_{\propto}=\left\{u \in\left[\jmath_{1}, \jmath_{2}\right]: u(\jmath)=\int_{\jmath_{1}}^{\jmath_{2}} G(\jmath, \wp) \mathbb{k}_{l}\left(\wp, \mathfrak{x}(\wp), \mathfrak{x}^{\prime}(\wp)\right) d \wp+\right.$ $\left.\lambda(\jmath) \geq \propto, \jmath \in[0, \Lambda], L_{l}(\mathfrak{x})(u) \geq \propto\right\}, l=1,2$,

$$
\left\{\begin{array}{l}
d(\mathfrak{x}, \mathfrak{y})=\max _{\jmath \in\left[\jmath_{1}, \jmath_{2}\right]}\left(\gamma\left|\Xi_{1} \mathfrak{x}(\jmath)-\Xi_{2} \mathfrak{y}(\jmath)\right|+\xi\left|\Xi_{1}^{\prime} \mathfrak{x}(\jmath)-\Xi_{2}^{\prime} \mathfrak{y}(\jmath)\right|\right) \sqrt{1+c^{2}} e^{i \tan c} \\
d\left(\left[L_{1} \mathfrak{x}\right]_{\propto}, \mathfrak{y}\right)=\max _{\jmath \in\left[\jmath_{1}, \jmath_{2}\right]}\left(\gamma\left|\Xi_{1} \mathfrak{x}(\jmath)-\Xi_{2} \mathfrak{y}(\jmath)\right|+\xi\left|\Xi_{1}^{\prime} \mathfrak{x}(\jmath)-\Xi_{2}^{\prime} \mathfrak{y}(\jmath)\right|\right) \sqrt{1+c^{2}} e^{i \tan c} \\
d\left(\left[L_{2} \mathfrak{y}\right]_{\propto}, \mathfrak{x}\right)=\max _{\jmath \in\left[\jmath_{1}, \jmath_{2}\right]}\left(\gamma\left|\Xi_{2} \mathfrak{y}(\jmath)-\Xi_{1} \mathfrak{x}(\jmath)\right|+\xi\left|\Xi_{2}^{\prime} \mathfrak{y}(\jmath)-\Xi_{1}^{\prime} \mathfrak{x}(\jmath)\right|\right) \sqrt{1+c^{2}} e^{i \tan c}
\end{array}\right\} .
$$

From assumption (4.3), we get for each $\jmath \in\left[\jmath_{1}, \jmath_{2}\right]$

$$
\begin{aligned}
\left|L_{1}(\mathfrak{x})(\jmath)-L_{2}(\mathfrak{y})(\jmath)\right|= & \left|\int_{\jmath_{1}}^{\jmath_{2}} G(\jmath, \wp)\right| \mathbb{k}_{1}\left(\wp, \mathfrak{x}(\wp), \mathfrak{x}^{\prime}(\wp)\right)-\mathbb{k}_{2}\left(\wp, \mathfrak{y}(\wp), \mathfrak{y}^{\prime}(\wp)\right)|| d \wp \\
\leq & \int_{\jmath_{1}}^{\jmath_{2}}|G(\jmath, \wp)| \mid \mathbb{k}_{1}\left(\wp, \mathfrak{x}(\wp), \mathfrak{x}^{\prime}(\wp)\right)-\mathbb{k}_{2}\left(\wp, \mathfrak{y}(\wp), w^{\prime}(\wp)\right) \| d \wp \\
\leq & \frac{\left(\jmath_{2}-\jmath_{1}\right)^{2}}{8} \delta_{1}\left(\max _{\jmath \in\left[\jmath_{1}, \jmath_{2}\right]} \mathcal{D}_{\mathfrak{x y}}(\jmath)\right) \mathcal{D}_{\mathfrak{x y}}(\jmath) \\
& +\delta_{2}\left(\max _{\jmath \in\left[\jmath_{1}, \jmath_{2}\right]} \mathcal{D}_{\mathfrak{x y}}(\jmath)\right) \mathcal{G}_{\mathfrak{x y}}(\jmath)
\end{aligned}
$$

and

$$
\begin{aligned}
\left|\left(L_{1}(\mathfrak{x})\right)^{\prime}(\jmath)-\left(L_{2}(\mathfrak{y})\right)^{\prime}(\jmath)\right|= & \left|\int_{\jmath_{1}}^{\jmath_{2}} G_{\jmath}(\jmath, \wp)\right| \mathbb{k}_{1}\left(\wp, \mathfrak{x}(\wp), \mathfrak{x}^{\prime}(\wp)\right)-\mathbb{k}_{2}\left(\wp, \mathfrak{y}(\wp), \mathfrak{y}^{\prime}(\wp)\right)|| d \wp \\
\leq & \int_{\jmath_{1}}^{\jmath_{2}}\left|G_{\jmath}(\jmath, \wp)\right| \mid \mathbb{k}_{1}\left(\wp, \mathfrak{x}(\wp), \mathfrak{x}^{\prime}(\wp)\right)-\mathbb{k}_{2}\left(\wp, \mathfrak{y}(\wp), \mathfrak{y}^{\prime}(\wp)\right) \| d \wp \\
\leq & \frac{\jmath_{2}-\jmath_{1}}{2} \delta_{1}\left(\max _{\jmath \in\left[\jmath_{1}, \jmath_{2}\right]} \mathcal{D}_{\mathfrak{x y}}(\jmath)\right) \mathcal{D}_{\mathfrak{x y}}(\jmath) \\
& +\delta_{2}\left(\max _{\jmath \in\left[\jmath_{1}, \jmath_{2}\right]} \mathcal{D}_{\mathfrak{x y}}(\jmath)\right) \mathcal{G}_{\mathfrak{x y}}(\jmath) .
\end{aligned}
$$


By virtue of (4.6) and (4.7), we conclude that

$$
\begin{aligned}
d\left(\left[L_{1}(\mathfrak{x})\right]_{\propto},\left[L_{2}(\mathfrak{y})(\jmath)\right]_{\propto}\right) \leq & \left(\gamma \frac{\left(\jmath_{2}-\jmath_{1}\right)^{2}}{8}+\xi \frac{\jmath_{1}-\jmath_{2}}{2}\right) \delta_{1}\left(\max _{\jmath \in\left[\jmath_{1}, \jmath_{2}\right]} \mathcal{D}_{\mathfrak{x y}}(\jmath)\right) \max _{\jmath \in\left[\jmath_{1}, \jmath_{2}\right]} \mathcal{D}_{\mathfrak{x y}}(\jmath) \\
& +\delta_{2}\left(\max _{\jmath \in\left[\jmath_{1}, \jmath_{2}\right]} \mathcal{D}_{\mathfrak{x y}}(\jmath)\right) \max _{\jmath \in\left[\jmath_{1}, \jmath_{2}\right]} \mathcal{G}_{\mathfrak{x y}}(\jmath) .
\end{aligned}
$$

It follows from (4.4) that

$$
\begin{aligned}
d\left(\left[L_{1}(\mathfrak{x})\right]_{\propto},\left[L_{2}(\mathfrak{y})(\jmath)\right]_{\propto}\right)< & \delta_{1}\left(\max _{\jmath \in\left[\jmath_{1}, \jmath_{2}\right]} \mathcal{D}_{\mathfrak{x y}}(\jmath)\right) \max _{\jmath \in\left[\jmath_{1}, \jmath_{2}\right]} \mathcal{D}_{\mathfrak{x y}}(\jmath) \\
& +\delta_{2}\left(\max _{\jmath \in\left[\jmath_{1}, \jmath_{2}\right]} \mathcal{D}_{\mathfrak{x y}}(\jmath)\right) \max _{\jmath \in\left[\jmath_{1}, \jmath_{2}\right]} \mathcal{G}_{\mathfrak{x y}}(\jmath),
\end{aligned}
$$

that is,

$$
\begin{aligned}
\delta_{1}\left(\max _{\jmath \in\left[\jmath_{1}, \jmath_{2}\right]} \mathcal{D}_{\mathfrak{x y}}(\jmath)\right) \max _{\jmath \in\left[\jmath_{1}, \jmath_{2}\right]} \mathcal{D}_{\mathfrak{x y}}(\jmath)+ & \delta_{2}\left(\max _{\jmath \in\left[\jmath_{1}, \jmath_{2}\right]} \mathcal{D}_{\mathfrak{x y}}(\jmath)\right) \max _{\jmath \in\left[\jmath_{1}, \jmath_{2}\right]} \mathcal{G}_{\mathfrak{x y}}(\jmath) \\
& \in s\left(d\left(\left[L_{1}(\mathfrak{x})\right]_{\propto},\left[L_{2}(\mathfrak{y})(\jmath)\right]_{\propto}\right)\right) .
\end{aligned}
$$

This implies that

$$
\delta_{1}(d(\mathfrak{x}, \mathfrak{y})) d(\mathfrak{x}, \mathfrak{y})+\delta_{2}(d(\mathfrak{x}, \mathfrak{y})) \frac{d\left(\left[L_{1} \mathfrak{x}\right]_{\propto}, \mathfrak{y}\right) d\left(\left[L_{2} \mathfrak{y}\right]_{\propto}, \mathfrak{x}\right)}{1+d(\mathfrak{x}, \mathfrak{y})} \in s\left(\left[L_{1} \mathfrak{x}\right]_{\propto},\left[L_{2} \mathfrak{y}\right]_{\propto}\right) .
$$

Therefore, an application of Corollary 3.4 implies that $L_{1}$ and $L_{2}$ have a common fixed point in $\mathcal{S}$, that is, (4.5) has a common solution. Consequently, in Theorem 4.2, if $L_{1}=$ $L_{2}=L$, then we can obtain integral equation (4.1) has a common solution in $\mathcal{S}$. Thus, the second-order nonlinear boundary value problem has a solution in $\mathcal{S}$.

Acknowledgment. The authors wish to thank the editors and anonymous referees for their useful comments and suggestions which have led to substantial improvements in the paper. This research is supported by NNSF of P. R. China (Grant No. 61503171), CPSF (Grant No. 2015M582091), NSF of Shandong Province (Grant No. ZR2016JL021), KRDP of Shandong Province (Grant No. 2017CXGC0701), DSRF of Linyi University (Grant No. LYDX2015BS001), and the AMEP of Linyi University, P. R. China.

\section{References}

[1] T. Allahviranloo, S. Salahshour, M. Homayoun-nejad, and D. Baleanu, General solutions of fully fuzzy linear systems, Abstr. Appl. Anal. 2013, 1-9, 2013.

[2] J. Ahmad, C. Klin-Eam, and A. Azam, Common fixed points for multivalued mappings in complex valued metric spaces with applications, Abstr. Appl. Anal. 2013, 1-12, 2013.

[3] A. Azam, Fuzzy fixed points of fuzzy mappings via a rational inequality, Hacet. J. Math. Stat. 40, 421-431, 2011.

[4] A. Azam and I. Beg, Common fuzzy fixed points for fuzzy mappings, Fixed Point Theory Appl. 2013, 1-11, 2013.

[5] A. Azam, B. Fisher, and M. Khan, Common fixed point theorems in complex valued metric spaces, Numer. Funct. Anal. Optim. 32, 243-253, 2011.

[6] A. Azam, N. Mehmood, M. Rashid, and S. Radenović, Fuzzy fixed point theorems in ordered cone metric spaces, Filomat 29, 887-896, 2015.

[7] S. Banach, Sur les opérations dans les ensembles abstraits et leur application aux équations intégrales, Fund. Math. 3, 133-181, 1922. 
[8] P. Balasubramaniam and S. Muralisankar, Existence and uniqueness of fuzzy solution for the nonlinear fuzzy integrodifferential equations, Appl. Math. Lett. 14, 455-462, 2001.

[9] Lj. B. Ćirić, A generalization of Banach's contraction principle, Proc. Amer. Math. Soc. 45, 267-273, 1974.

[10] B.K. Dass and S. Gupta, An extension of Banach contraction principle through rational expression, Indian J. Pure Appl. Math. 6, 1455-1458, 1975.

[11] T. Došenović, D. Rakić, B. Carić, and S. Radenović, Multivalued generalizations of fixed point results in fuzzy metric spaces, Nonlinear Anal. Model. Control 21, 211-222, 2016.

[12] V.D. Estruch and A. Vidal, A note on fixed fuzzy points for fuzzy mappings, Rend. Istit. Mat. Univ. Trieste 32, 39-45, 2001.

[13] X. Hao and L. Liu, Mild solution of semilinear impulsive integro-differential evolution equation in Banach spaces, Math. Methods Appl. Sci. 40, 4832-4841, 2017.

[14] X. Hao, M. Zuo, and L. Liu, Multiple positive solutions for a system of impulsive integral boundary value problems with sign-changing nonlinearities, Appl. Math. Lett. 82, 24-31, 2018.

[15] S. Heilpern, Fuzzy mappings and fixed point theorem, J. Math. Anal. Appl. 83, 566569, 1981.

[16] A. Jafarian, R. Jafari, A.K. Golmankhaneh, and D. Baleanu, Solving fully fuzzy polynomials using feed-back neural networks, Int. J. Comput. Math. 92, 742-755, 2015.

[17] V. Joshi, N. Singh, and D. Singh, $\phi$-contractive multivalued mappings in complex valued metric spaces and remarks on some recent papers, Cogent Math. 2016, 1-14, 2016.

[18] O. Kaleva, Fuzzy differential equations, Fuzzy Sets and Systems 24, 301-317, 1987.

[19] E. Karapinar and R.P. Agarwal, Further fixed point results on G-metric spaces, Fixed Point Theory Appl. 2013, 1-14, 2013.

[20] C. Klin-eam and C. Suanoom, Some common fixed-point theorems for generalizedcontractive-type mappings on complex-valued metric spaces, Abstr. Appl. Anal. 2013, $1-6,2013$.

[21] M.A. Kutbi, J. Ahmad, A. Azam, and A.S. Al-Rawashdeh, Generalized common fixed point results via greatest lower bound property, J. Appl. Math. 2014, 1-11, 2014.

[22] V. Lakshmikantham and R.N. Mohapatra, Theory of Fuzzy Differential Equations and Inclusions, Taylor \& Francis, Ltd., London, 2003.

[23] X. Liu, L. Liu, and Y. Wu, Existence of positive solutions for a singular nonlinear fractional differential equation with integral boundary conditions involving fractional derivatives, Bound. Value Probl. 2018, 1-21, 2018.

[24] D. Min, L. Liu, and Y. Wu, Uniqueness of positive solutions for the singular fractional differential equations involving integral boundary value conditions, Bound. Value Probl. 2018, 1-18, 2018.

[25] H.K. Nashine, C. Vetro, W. Kumam, and P. Kumam, Fixed point theorems for fuzzy mappings and applications to ordinary fuzzy differential equations, Adv. Difference Equ. 2014, 1-14, 2014.

[26] J.J. Nieto, The Cauchy problem for continuous fuzzy differential equations, Fuzzy Sets and Systems 102, 259-262, 1999.

[27] M.L. Puri and D.A. Ralescu, Fuzzy random variables, J. Math. Anal. Appl. 114, 409-422, 1986.

[28] S. Radenović and B.E. Rhoades, Fixed point theorem for two non-self mappings in cone metric spaces, Comput. Math. Appl. 57, 1701-1707, 2009.

[29] S. Radenović, P. Salimi, C. Vetro, and T. Došenović, Edelstein-Suzuki-type results for self-mappings in various abstract spaces with application to functional equations, Acta Math. Sci. Ser. B Engl. Ed. 36, 94-110, 2016. 
[30] S. Salahshour, A. Ahmadian, F. Ismail, and D. Baleanu, A novel weak fuzzy solution for fuzzy linear system, Entropy 18, 1-8, 2018.

[31] W. Shatanawi, V.Ć. Rajić, S. Radenović, and A. Al-Rawashdeh, MizoguchiTakahashi-type theorem in tvs-cone metric spaces, Fixed Point Theory Appl. 2012, 1-7, 2012.

[32] S. Seikkala, On the fuzzy initial value problem, Fuzzy Sets and Systems 24, 319-330, 1987.

[33] W. Sintunavarat, Y.J. Cho, and P. Kumam, Urysohn integral equations approach by common fixed points in complex-valued metric spaces, Adv. Difference Equ. 2013, 1-14, 2013.

[34] W. Sintunavarat and P. Kumam, Generalized common fixed point theorems in complex-valued metric spaces and applications, J. Inequal. Appl. 2012, 1-12, 2012.

[35] W. Sintunavarat, M.B. Zada, and M. Sarwar, Common solution of Urysohn integral equations with the help of common fixed point results in complex valued metric spaces, Rev. R. Acad. Cienc. Exactas Fís. Nat. Ser. A Mat. RACSAM 111, 531-545, 2017.

[36] S. Song, L. Guo, and C. Feng, Global existence of solutions to fuzzy differential equations, Fuzzy Sets and Systems 115, 371-376, 2000.

[37] D. Turkoglu and B.E. Rhoades, A fixed fuzzy point for fuzzy mapping in complete metric spaces, Math. Commun. 10, 115-121, 2005.

[38] Y. Yang and F. Meng, Existence of positive solution for impulsive boundary value problem with p-Laplacian in Banach spaces, Math. Methods Appl. Sci. 36, 650-658, 2013. 